\title{
Two New Calcium-rich Gap Transients in Group and Cluster Environments
}

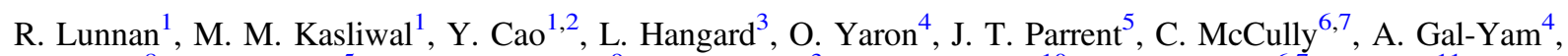 \\ J. S. Mulchaey ${ }^{8}$, S. Ben-Ami ${ }^{5}$, A. V. Filippenko ${ }^{9}$, C. Fremling ${ }^{3}$, A. S. Fruchter ${ }^{10}$, D. A. Howell ${ }^{6,7}$, J. Koda ${ }^{11}$, T. Kupfer ${ }^{1}$, \\ S. R. Kulkarni ${ }^{1}$, R. Laher ${ }^{12}$, F. Masci ${ }^{13}$, P. E. Nugent ${ }^{9,14}$, E. O. Ofek ${ }^{4}$, M. Yagi ${ }^{15}$, and Lin Yan ${ }^{13,16}$ \\ ${ }^{1}$ Department of Astronomy, California Institute of Technology, 1200 East California Boulevard, \\ Pasadena, CA 91125, USA; rlunnan@ astro.caltech.edu \\ 2 eScience Institute and Astronomy Department, University of Washington, Seattle, WA 98195, USA \\ ${ }^{3}$ Oskar Klein Centre, Physics Department, Stockholm University, SE-106 91 Stockholm, Sweden \\ ${ }^{4}$ Benoziyo Center for Astrophysics and the Helen Kimmel Center for Planetary Science, \\ Weizmann Institute of Science, 76100 Rehovot, Israel \\ ${ }^{5}$ Harvard-Smithsonian Center for Astrophysics, 60 Garden Street, Cambridge, MA 02138, USA \\ ${ }^{6}$ Las Cumbres Observatory Global Telescope Network, 6740 Cortona Drive, Suite 102, Goleta, CA 93117, USA \\ ${ }^{7}$ Department of Physics, University of California, Santa Barbara, Broida Hall, Mail Code 9530, Santa Barbara, CA 93106-9530, USA \\ ${ }_{9}^{8}$ The Observatories of the Carnegie Institution for Science, Pasadena, CA 91101, USA \\ ${ }^{9}$ Department of Astronomy, University of California, Berkeley, CA 94720-3411, USA \\ ${ }^{10}$ Space Telescope Science Institute, 3700 San Martin Drive, Baltimore, MD 21218, USA \\ ${ }^{11}$ Department of Physics and Astronomy, Stony Brook University, Stony Brook, NY 11794-3800, USA \\ ${ }^{12}$ Spitzer Science Center, California Institute of Technology, MS 314-6, Pasadena, CA 91125, USA \\ ${ }^{13}$ Infrared Processing and Analysis Center, California Institute of Technology, MS 100-22, Pasadena, CA 91125, USA \\ ${ }^{14}$ Lawrence Berkeley National Laboratory, 1 Cyclotron Road, MS 50B-4206, Berkeley, CA 94720, USA \\ 15 Optical and Infrared Astronomy Division, National Astronomical Observatory of Japan, 2-21-1 Osawa, Mitaka, Tokyo, 181-8588, Japan \\ ${ }_{16}$ Caltech Optical Observatories, California Institute of Technology, 1200 East California Boulevard, Pasadena, CA 91125, USA \\ Received 2016 November 23; revised 2016 December 20; accepted 2016 December 21; published 2017 February 8
}

\begin{abstract}
We present the Palomar Transient Factory discoveries and the photometric and spectroscopic observations of PTF11kmb and PTF12bho. We show that both transients have properties consistent with the class of calcium-rich gap transients, specifically lower peak luminosities and rapid evolution compared to ordinary supernovae, and a nebular spectrum dominated by [Ca II] emission. A striking feature of both transients is their host environments: PTF12bho is an intracluster transient in the Coma Cluster, while PTF11kmb is located in a loose galaxy group, at a physical offset $\sim 150 \mathrm{kpc}$ from the most likely host galaxy. Deep Subaru imaging of PTF12bho rules out an underlying host system to a limit of $M_{R}>-8.0 \mathrm{mag}$, while Hubble Space Telescope imaging of PTF11kmb reveals a marginal counterpart that, if real, could be either a background galaxy or a globular cluster. We show that the offset distribution of Ca-rich gap transients is significantly more extreme than that seen for SNe Ia or even short-hard gamma-ray bursts (sGRBs). Thus, if the offsets are caused by a kick, they require higher kick velocities and/or longer merger times than sGRBs. We also show that almost all Ca-rich transients found to date are in group and cluster environments with elliptical host galaxies, indicating a very old progenitor population; the remote locations could partially be explained by these environments having the largest fraction of stars in the intragroup/ intracluster light following galaxy-galaxy interactions.
\end{abstract}

Key words: supernovae: general - supernovae: individual (PTF11kmb, PTF12bho, PTF10hcw, SN 2005E)

Supporting material: machine-readable table

\section{Introduction}

In the past decade, large transient surveys have discovered a number of previously unknown classes of astrophysical transients, expanding the parameter space of known explosions, including several new classes in the former luminosity "gap" between supernovae (SNe) and novae (Kasliwal 2012). One such new class is the Ca-rich gap transients, with the peculiar transient SN 2005E as a prototype (Perets et al. 2010). Kasliwal et al. (2012; hereafter K12) proposed the following five properties to define the class: (1) intermediate luminosity ("gap" transients), (2) faster photometric evolution (rise and decline) than normal SNe, (3) photospheric velocities comparable to those of $\mathrm{SNe}$, (4) rapid evolution to the nebular phase, and (5) a nebular spectrum dominated by calcium emission. The rapid evolution indicates a class of transients with low ejecta masses $\left(\lesssim 0.5 M_{\odot}\right)$, whereas the nebular spectra suggest an ejecta composition dominated by calcium (Perets et al. 2010).
The sample of Ca-rich gap transients is still small. Following the discovery of the first such object, SN 2005E (Perets et al. 2010), the Palomar Transient Factory (PTF) (Law et al. 2009) presented three additional objects with similar properties: PTF09dav, PTF10iuv, and PTF11bij (Sullivan et al. 2011; K12). SN 2012hn, found by CRTS (Drake et al. 2009) and followed up by PESSTO, has since been added (Valenti et al. 2014). In addition, there are a number of archival candidates noted for their Ca-rich nebular spectra (Filippenko et al. 2003; Kawabata et al. 2010; Perets et al. 2010, 2011), from which K12 determined that SN 2007ke fits the Ca-rich gap transient criteria. The remainder of these archival candidates (six in total: SN 2000ds, SN 2001co, SN 2003H, SN 2003dg, SN 2003dr, and SN 2005cz) are sometimes referred to as "Ca-rich supernovae" (in particular, see Filippenko et al. 2003, who identified the first four such objects), but lack sufficient data to determine whether they constitute the same physical phenomenon as the more strictly defined Ca-rich gap transients. In particular, photometry is necessary to establish 
the low peak luminosity and low ejecta mass, and a high ratio of $\mathrm{Ca}$ to $\mathrm{O}$ in the nebular spectrum can also be seen in peculiar corecollapse and SNe Ia depending on the phase of the spectrum, and is in itself not sufficient (Pastorello et al. 2004; Valenti et al. 2009, 2014). For the purposes of this paper, we only consider the objects that satisfy both the photometric and spectroscopic criteria of K12, although we sometimes refer to the extended sample as candidates.

The progenitor system of Ca-rich gap transients is not known, but their host environments provide strong clues: out of the six members confirmed so far, only PTF09dav has a spiral host galaxy, while the rest have elliptical or S0 host galaxies, implying a predominantly old progenitor population. Even more striking, many are found at substantial offsets from their presumed host galaxies, suggesting either that they originate in extremely faint systems (such as globular clusters; Yuan et al. 2013), or alternatively that the progenitor has traveled from its birth site. Increasingly deep limits at the transient locations have failed to detect an underlying host system, favoring the latter interpretation (Lyman et al. 2013, 2014, 2016).

While the environments and locations strongly hint at an old progenitor population and thus a binary system progenitor, there are currently several proposed models for what the progenitor system actually is. Perets et al. (2010) suggested SN 2005E may be the result of He detonation from accretion from a He white dwarf onto a $\mathrm{CO}$ white dwarf, one of several variants of WD-WD progenitors proposed (Waldman et al. 2011; Sim et al. 2012; Dessart \& Hillier 2015). Another possibility is the tidal detonation of a He white dwarf by a neutron star or stellar-mass black hole (Metzger 2012; Sell et al. 2015). A better understanding of their origin is interesting not only from the point of view of binary evolution, but also from their nontrivial contribution to the $\mathrm{Ca}$ abundance: Mulchaey et al. (2014) showed that including the contribution from Ca-rich gap transients could resolve the discrepancy between the measured $\mathrm{Ca}$ abundances in galaxy clusters and the abundances expected from core-collapse and SN Ia yields. As the sample of Ca-rich transients is still small, adding to the number of well-studied objects is necessary. Here, we present the discovery of two new members of the class of Ca-rich gap transients discovered by the Palomar Transient Factory (PTF11kmb and PTF12bho), as well as an analysis of both the transients themselves and their host environments.

This paper is organized as follows. We present our observations of PTF11kmb and PTF12bho and their host environments in Section 2. The transient properties are given in Section 3, demonstrating that they are members of the class of Ca-rich gap transients. We discuss the host-galaxy properties in Section 4. The implications for the origins of Ca-rich gap transients and a summary are provided in Section 5 . We present data on an additional Ca-rich gap transient candidate, PTF10hcw, in the Appendix. All calculations in this paper assume a $\Lambda$ CDM cosmology with $H_{0}=70 \mathrm{~km} \mathrm{~s}^{-1} \mathrm{Mpc}^{-1}$, $\Omega_{M}=0.27$ and $\Omega_{\Lambda}=0.73$ (Komatsu et al. 2011).

\section{Observations}

\subsection{PTF Discoveries}

The objects PTF11 kmb and PTF12bho were found as part of the PTF (Law et al. 2009; Rau et al. 2009). PTF11kmb was discovered in data taken with the CFH12K 96-Megapixel camera (Rahmer et al. 2008; Law et al. 2010) mounted on the
48 inch Samuel Oschin Telescope at Palomar Observatory (P48) on 2011 August 16.25 (UT dates are used throughout this paper) at $\mathrm{J} 2000$ coordinates $\alpha=22^{\mathrm{h}} 22^{\mathrm{m}} 53.61, \delta=$ $+36^{\circ} 17^{\prime} 36^{\prime \prime}$. 5 , and at a magnitude $r=19.8 \mathrm{mag}$. A spectrum was taken with the Low Resolution Imaging Spectrometer (LRIS; Oke et al. 1995) on the $10 \mathrm{~m}$ Keck I telescope on 2011 August 28, showing SN features consistent with a SN Ib (see Filippenko 1997 for a review of SN spectral classification) at a redshift $z=0.017$ (Section 3.2), and was reported as such in the discovery telegram (Gal-Yam et al. 2011); subsequent follow-up observations revealed it to be a Ca-rich gap transient (see also Foley 2015). No host galaxy is seen underlying the $\mathrm{SN}$ position in the reference image.

The source PTF12bho was discovered in P48 data on 2012 February 25.25 at $\mathrm{J} 2000$ coordinates $\alpha=13^{\mathrm{h}} 01^{\mathrm{m}} 16^{\mathrm{s}} .65, \delta=$ $+28^{\circ} 01^{\prime} 18^{\prime \prime} .5$ and at a magnitude of $r=20.52$ mag. A spectrum taken with LRIS on 2012 March 15 yields $z=0.023$ based on the SN features (Section 3.2). Again, no underlying host galaxy is visible in the reference images; we note, however, that both the position and the redshift of PTF12bho are consistent with the Coma Cluster (Abell 1656).

\subsection{Light Curves}

We obtained $R$ - and $g$-band photometry of PTF $11 \mathrm{kmb}$ and PTF12bho with the P48 CFH12K camera. Additional followup photometry was conducted with the automated 60-inch telescope at Palomar (P60; Cenko et al. 2006) in the Bgri bands, and with the Las Cumbres Observatory (LCO) Faulkes Telescope North in gri (Brown et al. 2013). Host-subtracted point-spread function (PSF) photometry was obtained using the Palomar Transient Factory Image Differencing and Extraction (PTFIDE) pipeline (Masci et al. 2017) on the P48 images, and the pipeline presented by Fremling et al. (2016) on the P60 images. LCO data were processed using the tools available at github.com/logt/banzai, and PSF photometry was performed following Valenti et al. (2016). We correct the photometry for Galactic extinction following Schlafly \& Finkbeiner (2011), with $E(B-V)=0.092 \mathrm{mag}$ for PTF11kmb and $E(B-V)=0.008 \mathrm{mag}$ for PTF12bho. All photometric data are listed in Table 1, and are shown in Figures 1 and 2. PTF12bho was also observed with the Swift Ultra-Violet/ Optical Telescope (UVOT; Roming et al. 2005) and the Swift X-ray telescope (XRT; Burrows et al. 2005) on 2012 March 17.8 for $3 \mathrm{ks}$. We process the Swift data using the HEAsoft package $^{17}$ and find that PTF12bho is not detected with either instrument; the upper limit from UVOT is $u>22.95$ mag (AB mag, $3 \sigma)$. The XRT count limit is $<4.6 \times 10^{-3}$ counts $\mathrm{s}^{-1}$ $(3 \sigma)$; using the PIMMS tools ${ }^{18}$ we calculate a corresponding flux limit $F_{X}<1.6 \times 10^{-13} \mathrm{erg} \mathrm{cm}^{-2} \mathrm{~s}^{-1}$ if assuming a power-law source with index $\Gamma=2(0.3-10 \mathrm{keV})$.

\subsection{Spectroscopy}

We obtained a sequence of spectra for both PTF $11 \mathrm{kmb}$ and PTF12bho using LRIS on Keck I, the DEep Imaging MultiObject Spectrograph (DEIMOS; Faber et al. 2003) on the $10 \mathrm{~m}$ Keck II telescope, and the Double Spectrograph (DPSP; Oke \& Gunn 1982) on the 200-inch Hale telescope at Palomar Observatory (P200). The times of the spectra are marked as

\footnotetext{
17 http://heasarc.nasa.gov/lheasoft/

18 http://heasarc.gsfc.nasa.gov/cgi-bin/Tools/w3pimms/w3pimms.pl
} 
Table 1

Photometry of PTF11kmb and PTF12bho

\begin{tabular}{lccccc}
\hline \hline Object & $\begin{array}{c}\text { Observation } \\
\text { Date }\end{array}$ & $\begin{array}{c}\text { Phase } \\
\text { (rest- } \\
\text { frame } \\
\text { days) }\end{array}$ & Filter & Magnitude $^{\mathrm{a}}$ & Telescope \\
& (MJD) & (AB mag) & \\
\hline PTF11kmb & 55802.2 & 1.5 & $B$ & $19.84 \pm 0.09$ & P60 \\
PTF11kmb & 55805.2 & 4.5 & $B$ & $20.00 \pm 0.09$ & P60 \\
PTF11kmb & 55805.2 & 4.5 & $B$ & $20.11 \pm 0.10$ & P60 \\
PTF11kmb & 55808.2 & 7.5 & $B$ & $20.67 \pm 0.14$ & P60 \\
PTF11kmb & 55803.2 & 2.6 & $g$ & $19.47 \pm 0.03$ & P60 \\
PTF11kmb & 55806.2 & 5.5 & $g$ & $19.73 \pm 0.05$ & P60 \\
PTF11kmb & 55807.2 & 6.5 & $g$ & $19.84 \pm 0.06$ & P60 \\
PTF11kmb & 55808.2 & 7.5 & $g$ & $20.18 \pm 0.09$ & P60 \\
PTF11kmb & 55809.4 & 8.6 & $g$ & $20.14 \pm 0.09$ & P60 \\
\hline
\end{tabular}

Note.

${ }^{\mathrm{a}}$ Corrected for foreground extinction according to Schlafly \& Finkbeiner (2011).

(This table is available in its entirety in machine-readable form.)

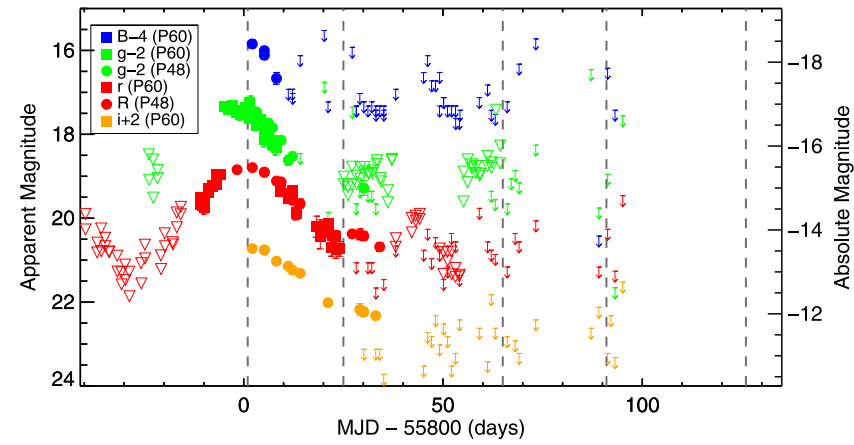

Figure 1. Observed light curves of PTF11kmb. Different filters are offset for clarity as indicated in the legend. The dashed lines mark the spectroscopy epochs. Upper limits are $5 \sigma$ and are shown as triangles (P48) or arrows (P60).

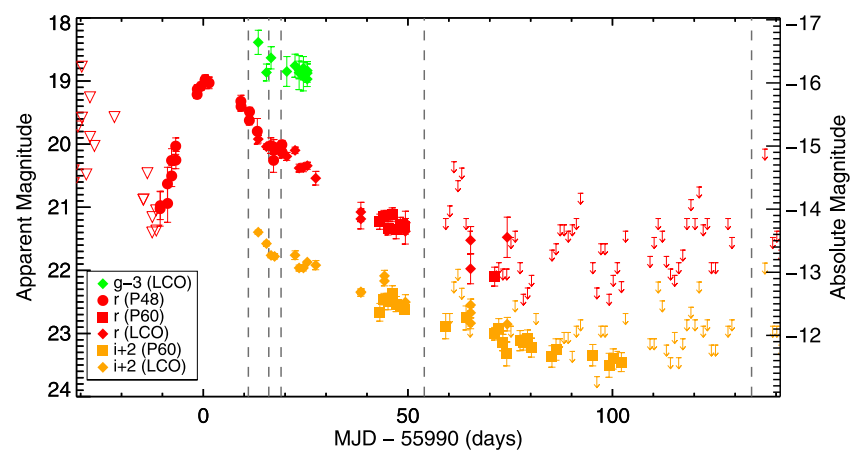

Figure 2. Observed light curves of PTF12bho. Different filters are offset for clarity as indicated in the legend. The dashed lines mark the spectroscopy epochs. Upper limits are $5 \sigma$ and are shown as triangles (P48) or arrows (P60).

dashed lines in Figures 1 and 2. Details of the spectroscopic observations are given in Table 2, and the spectroscopic sequences for PTF11 kmb and PTF12bho are shown in Figure 3. The spectroscopic properties are analyzed and discussed in Section 3.2.

\subsection{Host-galaxy Photometry}

We obtained deep imaging of the fields of PTF11 kmb using WFC3/UVIS on the Hubble Space Telescope (HST) through program GO-13864 (PI Kasliwal). This program also covered the field of SN 2005E (Perets et al. 2010), and we present and analyze the data on both transients here. Out of the Ca-rich gap transients confirmed so far, PTF $11 \mathrm{kmb}$ is the farthest from any apparent host, while SN 2005E is at the lowest redshift, making these two targets particularly interesting for searching for underlying faint sources. The details of the observations are listed in Table 3. The individual images were corrected for charge-transfer efficiency (CTE) losses using the tools available on the WFC3 website ${ }^{19}$, and combined using the standard Astrodrizzle package provided by STScI. Each image was drizzled to a final pixscale of 0 ! 02 pixel $^{-1}$ using a pixfrac of 0.8 .

While we do not have HST data covering PTF12bho, there is a wealth of other survey data available thanks to its location within the Coma Cluster. In particular, the position of PTF12bho is covered by the ultra-deep $R$-band SuprimeCam/Subaru data presented by Koda et al. (2015) and Yagi et al. (2016).

\subsection{Astrometry}

To determine the location of the transients relative to the deep HST and Subaru images, we first run SourceExtrac$\operatorname{tor}^{20}$ on both the deep host images and an image containing the transient, and construct catalogs of point sources in common between the two images. We then use IRAF's ccmap task to compute the astrometric tie between the two images and shift the SN image to the $H S T$ reference frame, achieving a combined astrometric uncertainty of 84 mas in the relative position of PTF11 kmb and 76 mas in the position of PTF12bho. For SN 2005E, there are not enough sources in common between the deep and small field of view HST image and the larger, shallower images containing the SN, so we use an image from the Sloan Digital Sky Survey (SDSS) as an intermediate step. The (combined) uncertainty in the astrometric tie for SN 2005E is 98 mas.

\subsection{Host-galaxy Spectroscopy}

The source PTF11 kmb is the Ca-rich transient discovered at the largest projected distance to the presumed host galaxy (NGC 7265) to date: $150 \mathrm{kpc}$. NGC 7265 is a member of a galaxy group, however, and several extended sources near PTF11kmb lack redshifts in NED. We therefore carried out two spectroscopic mask observations of the field around PTF $11 \mathrm{kmb}$ using DEIMOS on the Keck II telescope in order to assess whether any of the sources closer to PTF $11 \mathrm{kmb}$ were at a redshift consistent with the group and thus potential host galaxies at smaller offsets. The redshifts and coordinates of objects confirmed to be galaxies are listed in Table 4, and are discussed in Section 4.2.

\section{Two New Ca-rich Gap Transients: PTF11kmb and PTF12bho}

In this section, we analyze the properties of PTF $11 \mathrm{kmb}$ and PTF12bho, and show that they are members of the class of Carich gap transients.

\footnotetext{
19 http://www.stsci.edu/hst/wfc3/tools/cte_tools

${ }^{20}$ http://sextractor.sourceforge.net/
} 
Table 2

Summary of Spectroscopic Observations

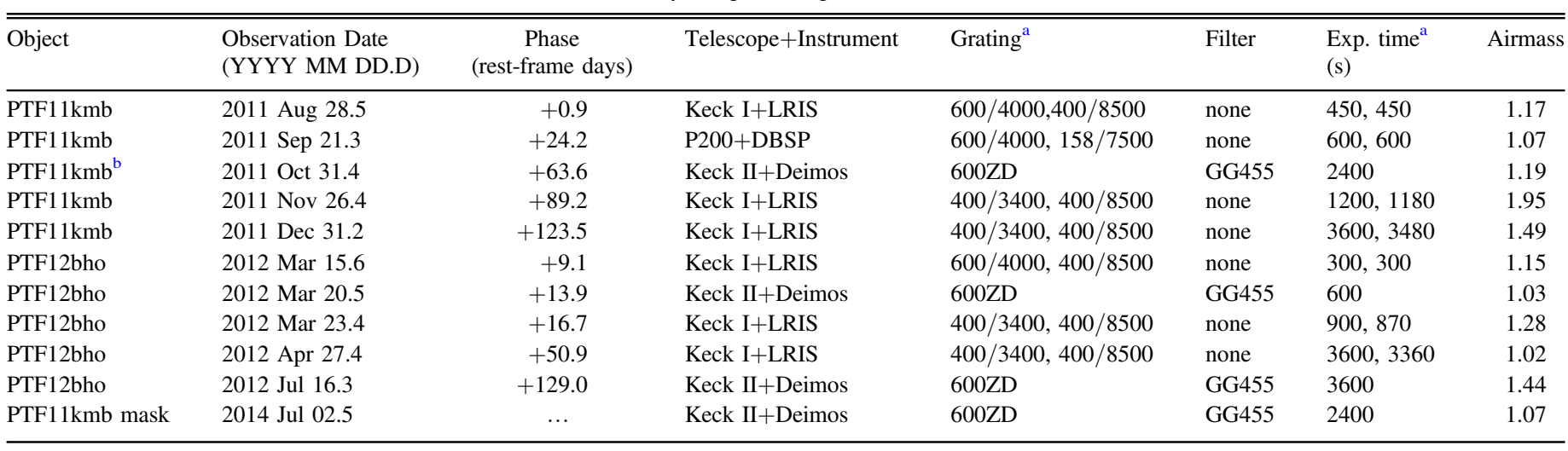

Notes.

a Comma-separated values indicate setup for blue and red arms, respectively.

b As part of a slitmask, aiming to obtain redshifts of potential host galaxies.

\subsection{Light-curve Properties}

The individual light curves of PTF11kmb and PTF12bho are shown in Figures 1 and 2, respectively. The peak luminosities of PTF11kmb and PTF12bho are $r=-15.5 \mathrm{mag}$ and $r=-16.0 \mathrm{mag}$ (respectively), comparable to previous Ca-rich gap transients. The $r$-band light curves are the best sampled, and we fit a low-order polynomial to the light curves near peak brightness to determine a best-fit time of peak: MJD 55784.9 for PTF11 kmb and MJD 55992.4 for PTF12bho. All phases reported are relative to these peak times.

The light-curve rise is best sampled for PTF12bho, where both pre-explosion limits as well as a $t^{2}$ fit to the rising portion of the light curve in flux space yield a rest-frame rise time to $r$ band maximum of 12 days. The rise is not quite as well constrained for PTF11 kmb owing to a gap in coverage before the first detection: the last upper limit is 18 days and the first detection 11 days before the peak; fitting a $t^{2}$ curve gives a restframe rise time of 15 days. We can use these measured rise times, as well as the velocity at peak determined from spectroscopy (Section 3.2.1), to make a simple estimate of the ejecta masses from $M_{\mathrm{ej}} \propto v t^{2}$ (Arnett 1982). Scaling from a normal SN Ia $\left(M_{\mathrm{ej}}=1.4 M_{\odot}, v=11,000 \mathrm{~km} \mathrm{~s}^{-1}, t_{\text {rise }}=17.4\right.$ days), we obtain approximate ejecta masses $M_{\mathrm{ej}} \approx 0.6-1.0 M_{\odot}$ for PTF $11 \mathrm{kmb}$ and $M_{\mathrm{ej}} \approx 0.4 M_{\odot}$ for PTF12bho.

Figure 4 shows the $r$-band light curves of PTF $11 \mathrm{kmb}$ and PTF12bho compared to the three previous PTF Ca-rich gap transients from K12. PTF11 kmb and PTF12bho have very similar light curves to the previous events in terms of both their peak luminosities and their rise and decline timescales. We therefore conclude that PTF11kmb and PTF12bho satisfy the light-curve criteria of K12 for Ca-rich gap transients: low peak luminosities and rapid photometric evolution.

\subsection{Spectroscopic Properties \\ 3.2.1. Photospheric-phase Spectra}

We show example photospheric-phase spectra of PTF11kmb and PTF12bho in Figure 5, compared to the Ca-rich gap transients from K12. PTF11 kmb was originally classified as a SN Ib (Gal-Yam et al. 2011) based on the strong He features in this spectrum. Helium features in the photospheric spectrum are a common (if not defining) property of Ca-rich gap transients, and were also seen in SN 2005E, PTF10iuv, and SN 2007ke. Using the minimum of the He lines, we measure a photospheric velocity of $\sim 11,000 \mathrm{~km} \mathrm{~s}^{-1}$ for PTF11 kmb.

The photospheric spectrum of PTF12bho is more complex and warrants further investigation. We use the spectral synthesis code SYN++ (Thomas et al. 2011) to identify as many of the lines as possible and to obtain an estimate of the photospheric expansion velocity. SYN++ calculates a synthetic spectrum by considering an optically thick pseudo-photosphere surrounded by an extended line-forming region using a number of simplifying assumptions including spherical symmetry, local thermodynamic equilibrium (LTE), and only thermal excitations. The fit is constrained by matching both the absorption profiles and the relative strength of different features.

Figure 6 shows the resulting SYN++ fit for the +9 day PTF12bho spectrum, with the main features marked. The fit shown has a blackbody temperature of $4500 \mathrm{~K}$ and velocities in the range of $6000-10,000 \mathrm{~km} \mathrm{~s}^{-1}$. We consider Ca II, O I, $\mathrm{Mg}$ II, Fe II, and He I as reasonably secure identifications based on multiple lines of the same species seen in the spectrum. The relative line strengths of $\mathrm{He}$ I are not perfectly reproduced, but this is expected as it would require non-LTE calculations, which are not included in SYN++ (e.g., Lucy 1991; Baron et al. 1996; Branch et al. 2002).

A number of other species were attempted and can neither be ruled out nor securely identified, as they generally produce only one strong feature in the spectrum. These possible species include C II, O II, and Ca I. Both Co II and Ti II are possible, and mainly contribute in bringing the flux down in the blue. Al II can match the feature at $6780 \AA$, but only if moving at $12,000 \mathrm{~km} \mathrm{~s}^{-1}$, significantly above the photospheric velocity. Sc II (which was seen in PTF09dav; Sullivan et al. 2011) was considered for the feature at $5540 \AA$, but the corresponding feature at $6050 \AA$ is not well matched, and the expected strong line at $5400 \AA$ is not seen in the spectrum. Interestingly, we also cannot rule out $\mathrm{HI}$, which matches the feature at $6350 \AA$ if moving at a velocity of $\sim 10,000 \mathrm{~km} \mathrm{~s}^{-1}$, comparable to other species in the fit.

A number of features are still unidentified $(5540 \AA$, $6640 \AA$, $6780 \AA, 6700 \AA, 7430 \AA, 8080-8220 \AA, 8770 \AA, 9400 \AA)$. The failure of SYN++ to find matches for these features is likely due to a combination of factors: problems with the (LTE) line strengths assumed in the model, forbidden lines, or lines moving at significantly higher velocities than the photosphere. 

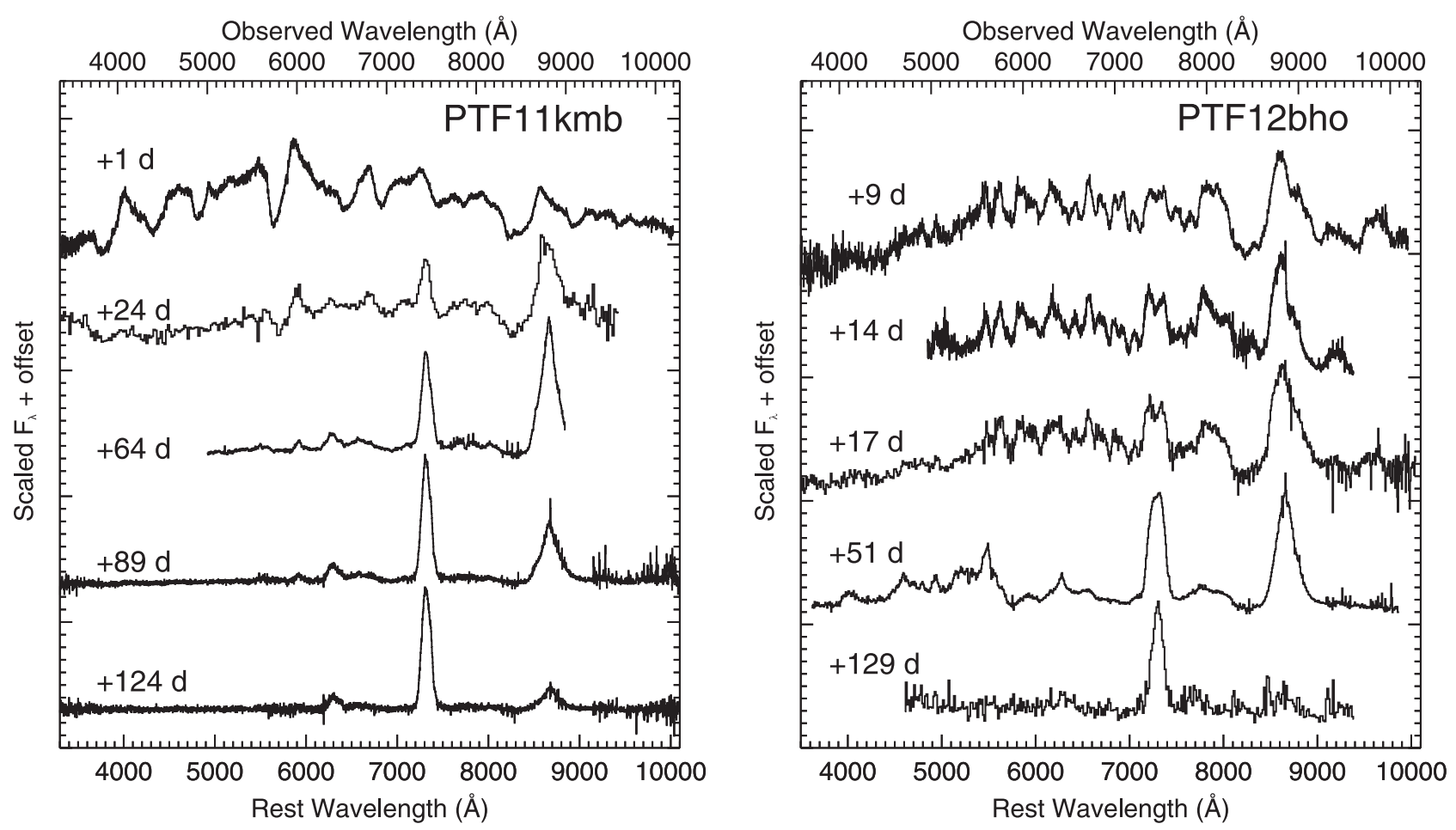

Figure 3. Observed spectroscopic sequence of PTF11 kmb (left) and PTF12bho (right). The phase of each spectrum with respect to the $r$-band maximum is marked. Note the early transition to the nebular phase, and the high ratio of [Ca II] $\lambda \lambda 7291,7324$ compared to [O I] $\lambda \lambda 6300,6364$.

Table 3

Summary of HST Observations

\begin{tabular}{llllc}
\hline \hline Object & $\begin{array}{l}\text { Observation Date } \\
\text { (YYYY }\end{array}$ & Instrument & Filter & $\begin{array}{c}\text { Total } \\
\text { Exp. Time }\end{array}$ \\
& MM DD) & & & $(\mathrm{s})$ \\
\hline PTF11kmb & 2015 Jul 12 & WFC3/UVIS & F606W & 5469 \\
SN 2005E & 2014 Dec 10 & WFC3/UVIS & F606W & 5361 \\
\hline
\end{tabular}

The uncertainty in the precise redshift of PTF12bho owing to the lack of an unambiguously identified host galaxy also adds to the difficulty. While a more thorough exploration of the spectral sequence of PTF12bho would be very interesting for shedding light on this object, we consider it to be beyond the scope of this paper.

We note that when $\mathrm{K} 12$ were attempting to empirically define the class of Ca-rich gap transients, they allowed for diversity in the photospheric-phase spectra, and did not (for example) require the detection of He. Without this allowance, an object like PTF09dav, which was originally reported as a subluminous SN Ia, would not have been included (Sullivan et al. 2011). PTF12bho adds to this diversity, with a photospheric spectrum that is unique among the Ca-rich gap transients discovered to date. Understanding this diversity will be important in determining the physical phenomenon giving rise to the Carich gap transients, including whether objects like PTF09dav and PTF12bho are truly part of the same class of objects.

\subsubsection{Nebular-phase Spectra}

As with other Ca-rich gap transients, PTF11kmb and PTF12bho transition to the nebular phase on relatively short timescales. A +51 day spectrum of PTF12bho and a +89 day spectrum of PTF11kmb are shown in Figure 7, again compared to nebular spectra of other members of this class. While the PTF12bho spectrum still exhibits some photospheric features (which was also the case for SN 2005E at a comparable phase), both spectra are dominated by nebular Ca II emission. We note in particular the strong forbidden [Ca II] $\lambda \lambda 7291,7324$ emission line and the comparatively weak [O I] $\lambda \lambda 6300,6363$ emission. This is one of the defining characteristics of the Ca-rich gap transient class-while other SN types may also show strong [Ca II] emission, the ratio of [Ca II] to [O I] is typically much lower (see e.g., Figure 4 of K12).

We also note that at the phases displayed here, PTF $11 \mathrm{kmb}$ and PTF12bho also exhibit strong emission in the permitted Ca II triplet-our series of spectra (Figure 3) show that the strength of this line decreases with time, while the forbidden [Ca II] line stays strong compared to [O I] in all of our nebular spectra. Using the strength of [CaII] relative to [O I] as one of the defining characteristics of Ca-rich gap transients therefore does not seem to depend strongly on the exact phase of the spectrum.

Combining the photometric and spectroscopic information, then, we have shown that PTF11 kmb and PTF12bho both satisfy the five characteristics of Ca-rich gap transients: (1) low peak luminosities, (2) rapid photometric evolution, (3) normal photospheric-phase velocities, (4) early evolution to the nebular phase, and (5) nebular spectra with a high ratio of $\mathrm{Ca}$ compared to $\mathrm{O}$. Thus we conclude that both objects are unambiguous members of the class of Ca-rich gap transients as described by K12.

\section{Galaxy Environments of Ca-Rich Gap Transients}

A peculiar feature of Ca-rich gap transients is that they tend to be found at large offsets from any apparent host (K12). This is true also of PTF11kmb and PTF12bho: no underlying host is visible in the PTF reference images for either transient. PTF11kmb stands out in particular from being at least $80 \mathrm{kpc}$ 
Table 4

Redshifts of Galaxies Near PTF11kmb

\begin{tabular}{|c|c|c|c|c|c|}
\hline Galaxy ID & $\alpha(\mathrm{J} 2000)$ & $\delta(\mathrm{J} 2000)$ & Redshift & In Group? & Telescope/Instrument \\
\hline 2MASX J22223308+3616504 & $22^{\mathrm{h}} 22^{\mathrm{m}} 33^{\mathrm{s}} \cdot 08$ & $+36^{\circ} 16^{\prime} 50^{\prime \prime} 4$ & 0.0179 & Yes & Keck II/Deimos \\
\hline GALEXASC J222249.41+361811.7 & $22^{\mathrm{h}} 22^{\mathrm{m}} 49^{\mathrm{s}} \cdot 41$ & $+36^{\circ} 18^{\prime} 11^{\prime \prime} 7$ & 0.02099 & Yes & P200/DBSP \\
\hline GALEXASC J222226.82+362011.9 & $22^{\mathrm{h}} 22^{\mathrm{m}} 26^{\mathrm{s}} \cdot 82$ & $+36^{\circ} 20^{\prime} 11^{\prime \prime} \cdot 9$ & 0.166 & No & Keck II/Deimos \\
\hline (anon.) & $22^{\mathrm{h}} 22^{\mathrm{m}} 52^{\mathrm{s}} \cdot 30$ & $+36^{\circ} 17^{\prime} 39^{\prime \prime} .9$ & 0.166 & No & Keck II/Deimos \\
\hline (anon.) & $22^{\mathrm{h}} 22^{\mathrm{m}} 37^{\mathrm{s}} .03$ & $+36^{\circ} 20^{\prime} 43^{\prime \prime} 6$ & 0.166 & No & Keck II/Deimos \\
\hline (anon.) & $22^{\mathrm{h}} 22^{\mathrm{m}} 42^{\mathrm{s}} \cdot 66$ & $+36^{\circ} 19^{\prime} 25^{\prime \prime} 3$ & 0.166 & No & Keck II/Deimos \\
\hline (anon.) & $22^{\mathrm{h}} 23^{\mathrm{m}} 05^{\mathrm{s}} \cdot 50$ & $+36^{\circ} 19^{\prime} 00^{\prime \prime} .5$ & 0.403 & No & Keck II/Deimos \\
\hline
\end{tabular}

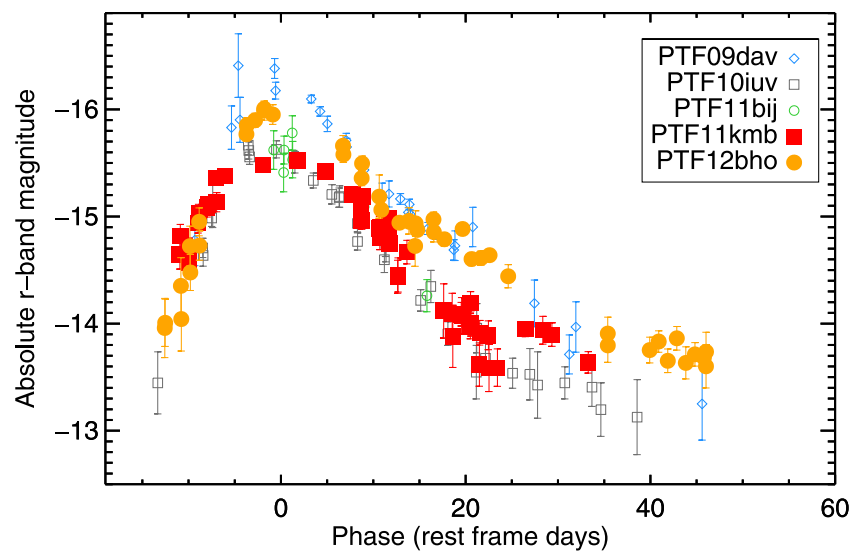

Figure 4. The $r$-band light curves of PTF11 kmb and PTF12bho compared to the Ca-rich gap transients from PTF presented by K12. PTF11kmb and PTF12bho have light curves that are very similar to the previous objects, with relatively low luminosities and short rise times compared to typical SNe.

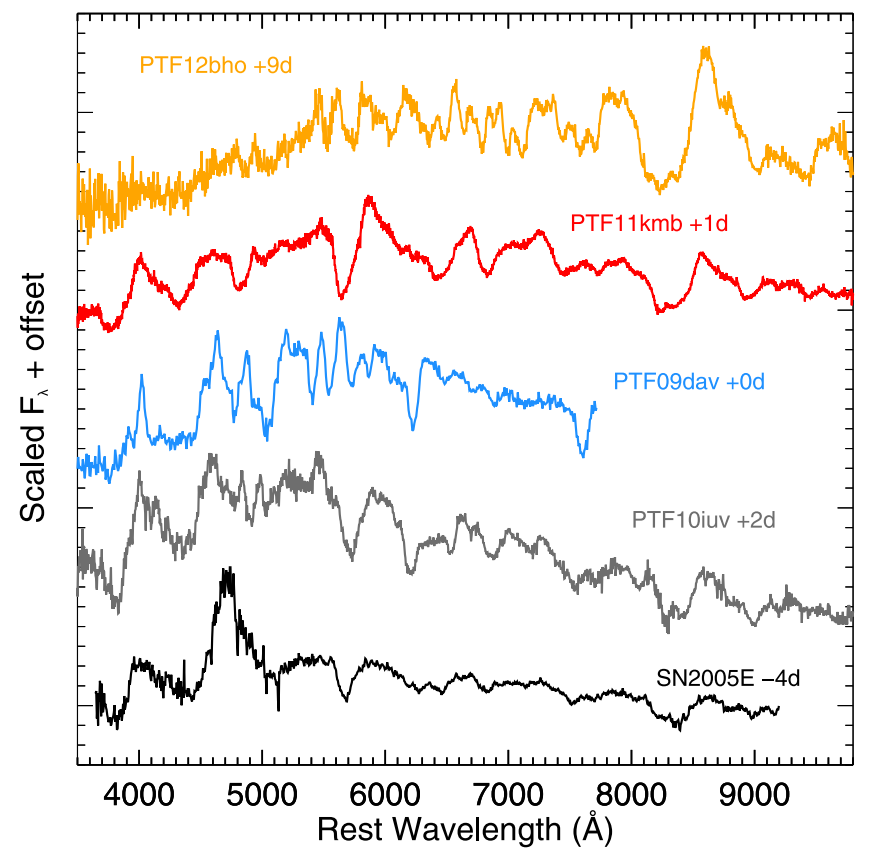

Figure 5. Photospheric-phase spectra of PTF11kmb and PTF12bho, compared to other Ca-rich gap transients. The photospheric-phase spectra of this class are diverse, although they generally show velocities comparable to those of other $\mathrm{SNe}$. Many exhibit He features - the photospheric spectrum of PTF $11 \mathrm{kmb}$ is an excellent match to that of a SN Ib. The spectrum of PTF12bho is very complex, and we analyze it in more detail in Figure 6.

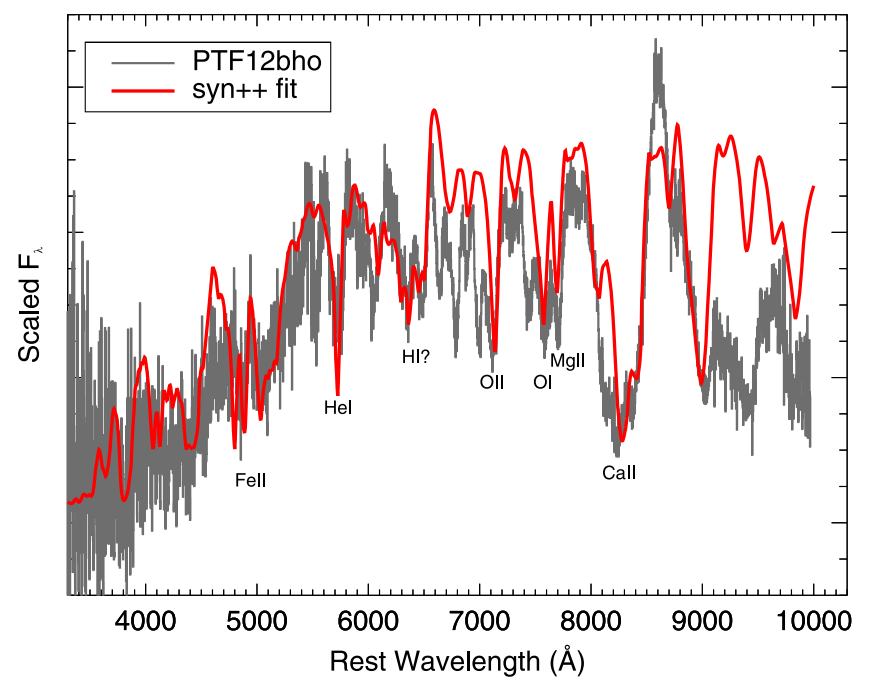

Figure 6. SYN ++ fit to the +9 day spectrum of PTF12bho. The spectrum is very complex and a number of features are still unidentified. The main species we can securely identify are Ca II, O I, Fe II, Mg II, and He I.

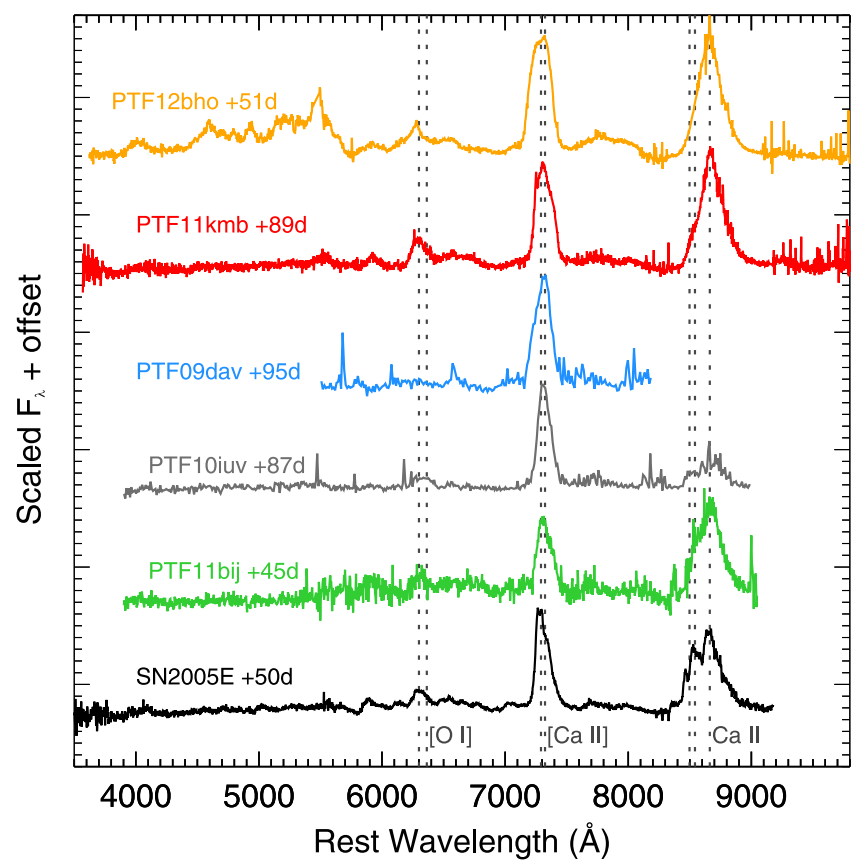

Figure 7. Nebular-phase spectra of PTF11kmb and PTF12bho, compared to those of other Ca-rich gap transients. PTF12bho shows a mix of photospheric and nebular features in its spectrum at 52 days past peak; the same was true for SN 2005E. Note the high ratio of $\mathrm{Ca}$ to O, particularly the strong [Ca II] $\lambda \lambda 7291,7324$ emission line, which is characteristic of this class of transient. 

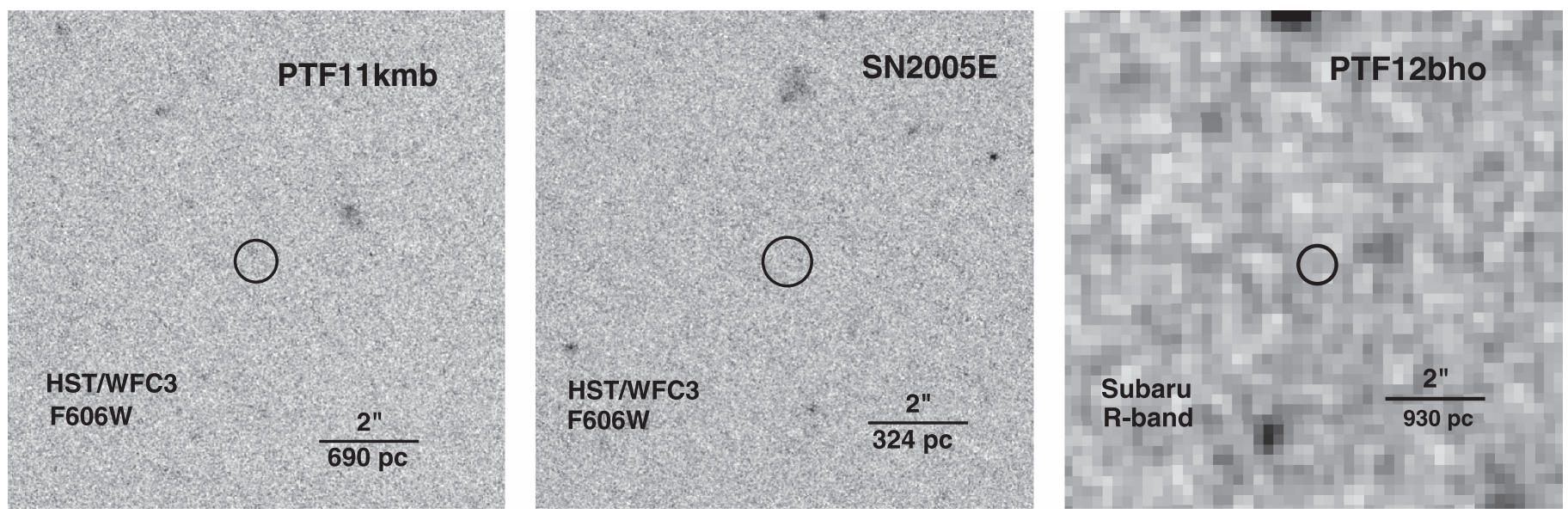

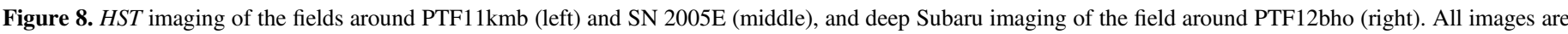

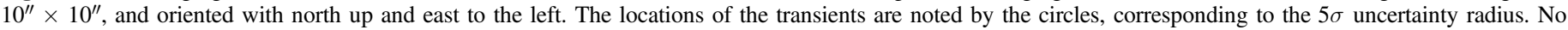
sources are detected at the positions of SN 2005E and PTF12bho, while there is a marginally detected source within the uncertainty radius of PTF11kmb.

from the closest host candidate with a redshift in NED. The closest galaxy to PTF12bho is SDSS J130109.43+280159.2, at a projected distance of $48 \mathrm{kpc}$. In this section, we analyze the host environments of PTF11 kmb and PTF12bho in detail, and discuss them in the context of the sample of Ca-rich gap transients.

\subsection{Limits on In Situ Formation}

As described in Section 2.4, we have deep imaging of the locations of PTF $11 \mathrm{kmb}$ and SN 2005E from HST, and of PTF12bho from Subaru. The images at the transient positions are shown in Figure 8 . Each cutout is $10^{\prime \prime} \times 10^{\prime \prime}$, and the circles correspond to the $5 \sigma$ uncertainty radius in the transient position from the astrometric tie (Section 2.5). No underlying sources are seen at the positions of SN 2005E or PTF12bho, to upper limits $m_{606 \mathrm{~W}}>28.9 \mathrm{mag}$ and $m_{R}>27.0 \mathrm{mag}$, respectively. The upper limit for SN 2005E corresponds to an absolute magnitude $M_{606 \mathrm{~W}}>-4.0 \mathrm{mag}$, thus ruling out the majority of the globular cluster luminosity function (Jordán et al. 2007); for PTF12bho the upper limit corresponds to an absolute magnitude $M_{r}>-8.0$ mag. Similarly deep limits also exist for the Carich gap transients SN 2012hn $\left(M_{R}>-5.6 \mathrm{mag}\right.$; Lyman et al. 2014) and SN 2007ke $\left(M_{606 \mathrm{~W}}>-6.6 \mathrm{mag}\right.$; Lyman et al. 2016), indicating that the majority of Ca-rich gap transients likely did not form at their explosion sites.

The case of PTF $11 \mathrm{kmb}$ is slightly more complicated: here, there is a marginally significant source within the $5 \sigma$ position error circle. Aperture photometry yields an apparent magnitude $m=28.3 \pm 0.15 \mathrm{mag}$. If at the same redshift as PTF $11 \mathrm{kmb}$, this corresponds to an absolute magnitude of $-6.0 \mathrm{mag}$, which would be consistent with a globular cluster, although on the faint end of the luminosity function. The putative source is too faint for spectroscopy to assess whether it is at the same redshift as PTF $11 \mathrm{kmb}$, and other similarly faint features in the image appear streak-like and are most likely artifacts produced by CTE correction problems. Deeper imaging, as well as imaging in multiple filters, can establish whether the marginal source is real, and whether its colors are best explained by a globular cluster or a background/foreground source.

We also caution that at these faint magnitudes, the sky density of background sources is high. Following Berger (2010) and Bloom et al. (2002), based on the source density distribution observed in surveys like the Hubble Ultra Deep
Field (Beckwith et al. 2006), the probability of a 28.3 mag source within our $5 \sigma$ uncertainty radius being a random background galaxy is about $7 \%$. While the source appears to be extended, the signal-to-noise ratio is low, so we cannot completely rule out a foreground star, although we consider it less likely: for a source this faint to be within the Galaxy, it would have to be either a late-M dwarf or a white dwarf (absolute magnitude 11-12) far out in the halo. Given the marginal nature of the source and the relatively high likelihood of chance coincidence, we conclude that a globular cluster origin for PTF11kmb cannot be ruled out.

\subsection{Offset Distribution}

Identifying the true host galaxies of either PTF $11 \mathrm{kmb}$ or PTF12bho is ambiguous. Foley (2015) argued that NGC 7265 is the most likely host of PTF11kmb: while offset by $\sim 150 \mathrm{kpc}$ in physical units, it is the closest galaxy in terms of isophotal radii and the closest in redshift to $\mathrm{PTF} 11 \mathrm{kmb}$ out of the galaxies with a redshift in NED. However, our spectroscopy shows that there are at least two more galaxies that are likely associated with the galaxy group and with smaller projected offsets: GALEXASC J222249.41+361811.7 at $1^{\prime}$ northwest and $z=0.021$, labeled " $\mathrm{A}$ " in Figure 9, and 2MASX J22223308+3616504 at 4!2 west-southwest at $z=$ 0.0179 , labeled "B." We note that unlike the other nearby group galaxies, GALEXASC J222249.41+361811.7 exhibits strong $\mathrm{H} \alpha$ emission, indicating ongoing star formation. Importantly, no galaxy at a consistent redshift with PTF11 kmb was found closer than $1^{\prime}$-while a source 16 "' 2 west of PTF11 kmb was revealed to be a galaxy (circled in red), we find that it is background at $z=0.166$, and therefore unrelated to the transient.

GALEXASC J222249.41+361811.7 ("A") is the closest potential host galaxy to PTF $11 \mathrm{kmb}$ in terms of both projected distance and host-normalized projected distance $(21 \mathrm{kpc}$ and $\sim 16$ galaxy half-light radii, respectively), although it is offset by $\sim 1200 \mathrm{~km} \mathrm{~s}^{-1}$ from the redshift of $z=0.017$ derived from the SN spectrum. NGC 7265 at $150 \mathrm{kpc}$ is the second closest in terms of host-normalized distance ( $\sim 20$ half-light radii), and at a redshift consistent with that derived from the $\mathrm{SN}$ $(z=0.0169)$. The galaxies labeled " $\mathrm{B}$ " and " $\mathrm{C}$ " in Figure 9 are closer than NGC7265 in terms of projected distance, 


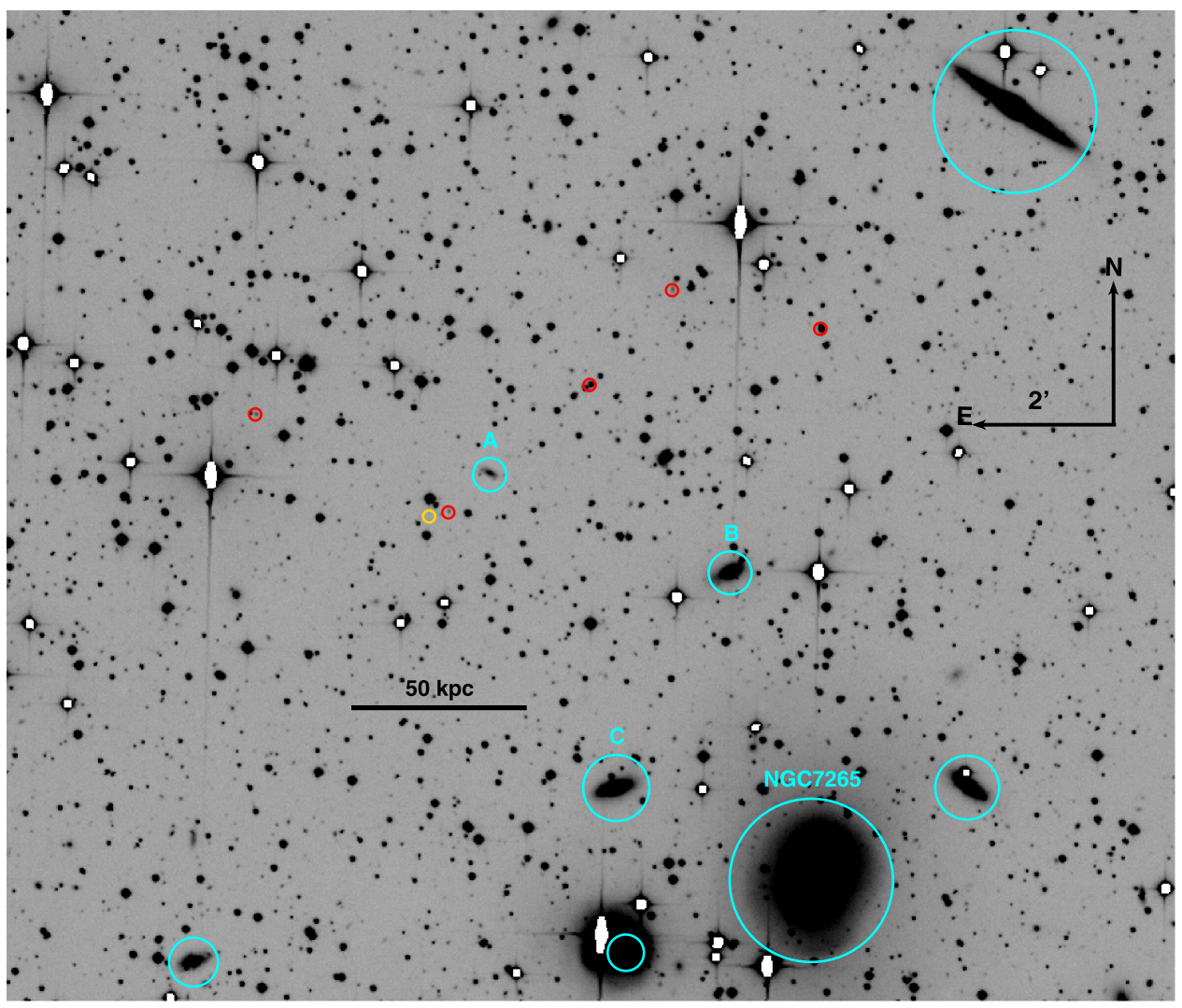

Figure 9. PTF $R$-band reference image of the field around PTF11kmb. The yellow circle marks the position of the transient. Objects circled in cyan are galaxies with redshifts consistent with the galaxy group. The red circles mark objects that were found to be background galaxies from our spectroscopic mask. All other objects identified from the mask turned out to be foreground stars.
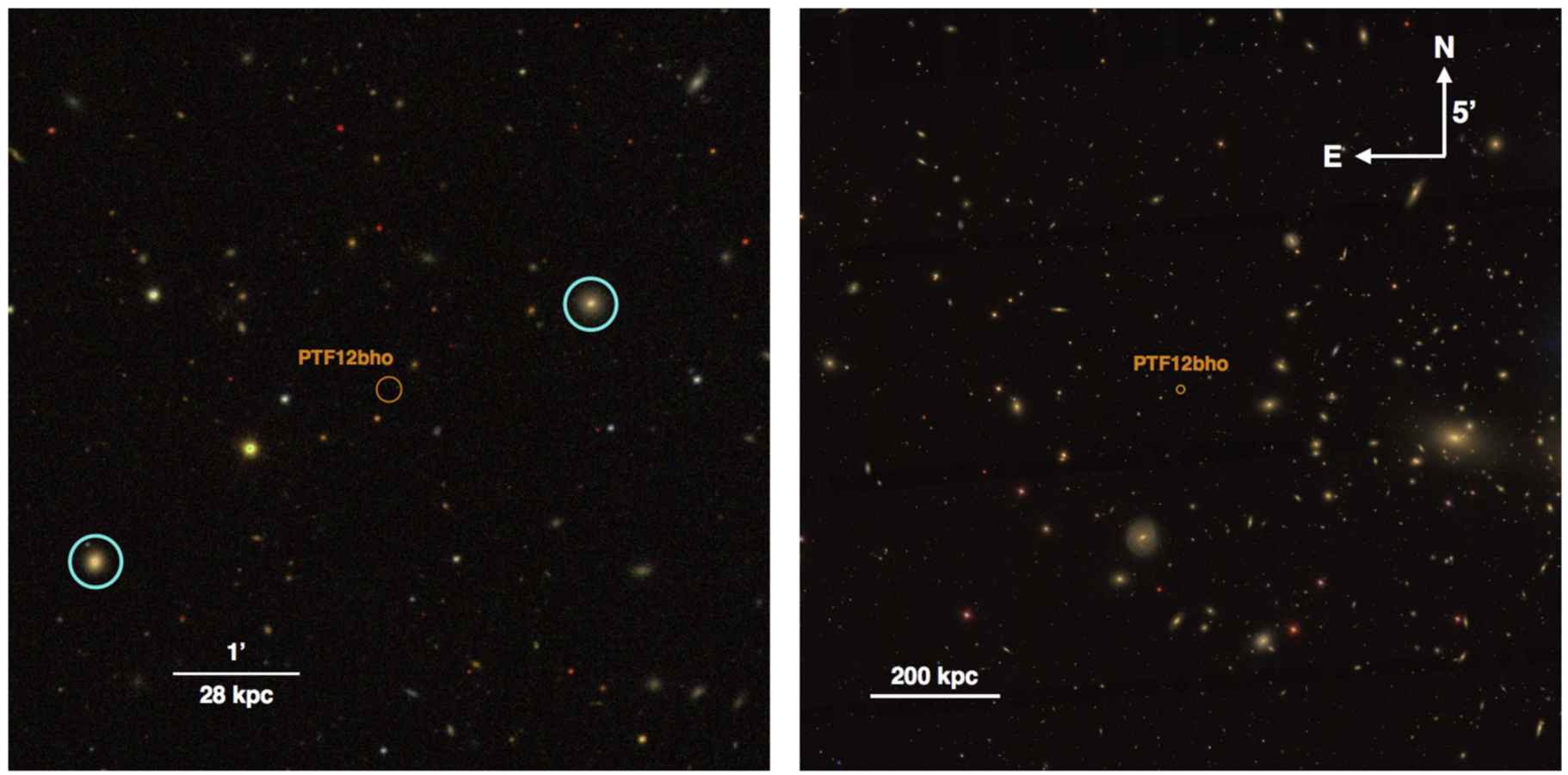

Figure 10. Left: $6^{\prime} \times 6^{\prime}$ SDSS gri composite image of the field around PTF12bho. The two closest galaxies having redshifts consistent with the Coma Cluster are circled. Right: $42^{\prime} \times 42^{\prime}$ wide-field view showing the position of PTF12bho within the Coma Cluster.

although not in host-normalized distance. For the purposes of measuring offsets, we assume NGC7265 is the host of PTF11 kmb as it is closer in redshift and offset a similar hostnormalized distance compared to GALEXASC J222249.41
+361811.7 . We note that our results are not significantly influenced by this choice, however: either way, PTF $11 \mathrm{kmb}$ is significantly offset both in physical and host-normalized units, and the statistics comparing Ca-rich gap transients to other 

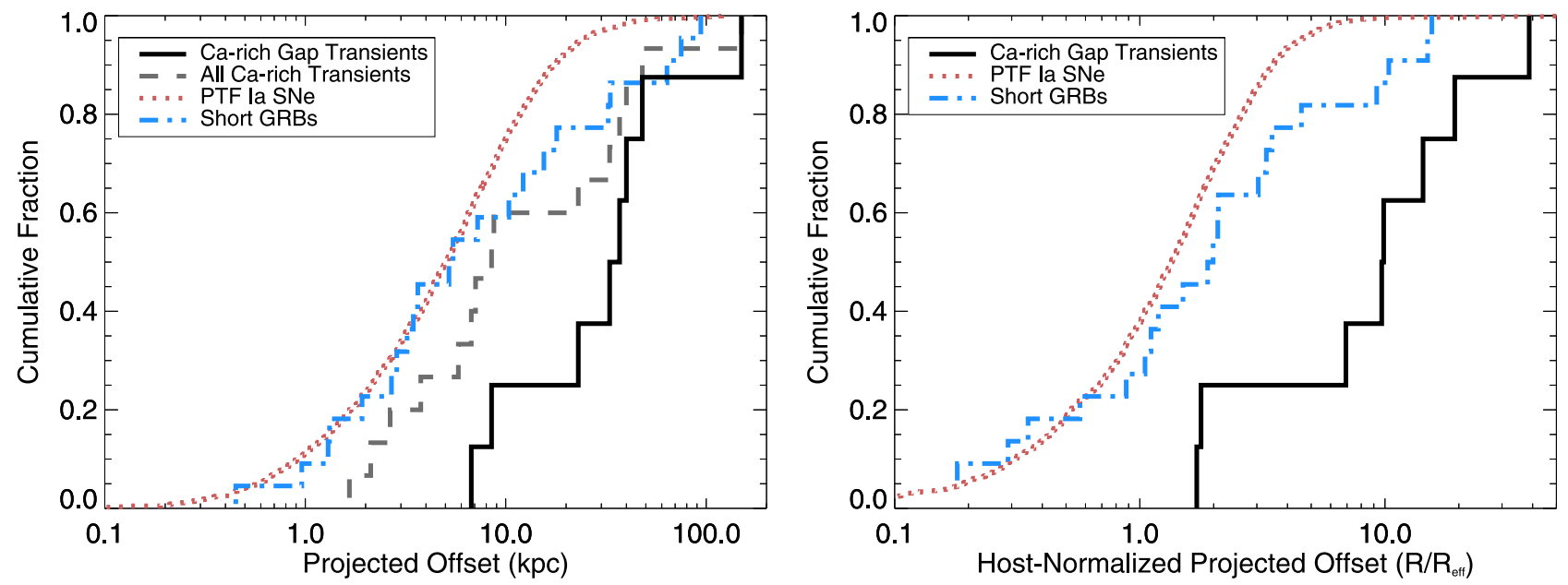

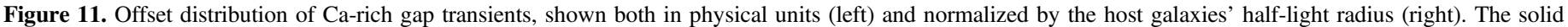

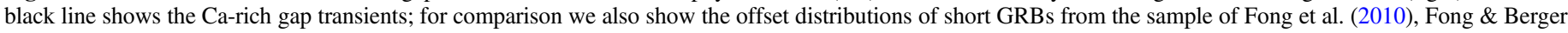

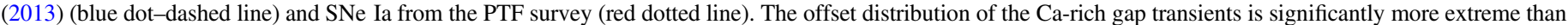

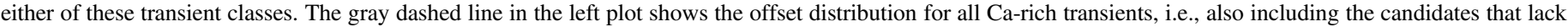

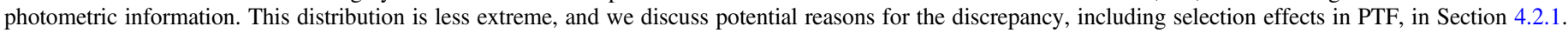

populations are not affected by instead adopting GALEXASC J222249.41+361811.7 as the host.

Similarly, PTF12bho is $1.73 \quad(48 \mathrm{kpc})$ away from SDSS J130109.43+280159.1, the nearest galaxy with a measured redshift, and which we nominally adopt as the host. On the other hand, being located in the intracluster medium of the Coma Cluster, there are a number of potential host galaxies at a compatible redshift. Figure 10 shows the field around PTF12bho with the two closest galaxies with compatible redshifts circled (left), as well as a zoom-out of the field showing PTF12bho's location within the Coma Cluster (right).

With these host associations, Figure 11 shows the resulting offset distribution for the known Ca-rich gap transients compared to two other transient populations believed to have old binary progenitors: SNe Ia (showing the PTF sample; L. Hangard et al. 2017, in preparation), and short-duration gamma-ray bursts (sGRBs; Fong et al. 2010; Fong \& Berger 2013). The left panel displays the physical projected offsets, while the right shows offsets normalized by the hosts' half-light radii. In either view, the offset distribution of Ca-rich gap transients is extreme, with all of the objects in the confirmed sample found at offsets greater than one half-light radius. The differences with both $\mathrm{SNe}$ Ia and sGRBs are statistically significant: $p=0.0004$ and $p=0.04$ (respectively), as determined by a Kolmogorov-Smirnov test.

\subsubsection{Survey Selection Effects}

It is potentially worrying that all of the PTF Ca-rich gap transients to date have been found at offsets $>20 \mathrm{kpc}$, while all the (candidate) Ca-rich gap transients from other surveys were found at offsets $<10 \mathrm{kpc}$ with the exception of SN 2005E at $23 \mathrm{kpc}$. This is also illustrated by the gray line in Figure 11, which shows the distribution of offsets including the candidate Ca-rich transients that lack light-curve information. The best way to quantify the extent to which PTF's detection efficiency for fast and faint transients is affected by the underlying galaxy surface brightness would be to inject fake transients and run a recovery analysis. Such an analysis is beyond the scope of this paper, but will be presented by C. Frohmaier et al. (2017, in preparation).
As a simpler test of whether PTF is missing a large fraction of faint transients on top of galaxies, we search the PTF database for all objects that were classified as having "gap" luminosities between 2009 and 2012. We find 17 such objects in total, including the 5 Ca-rich gap transients PTF09dav, PTF10iuv, PTF11bij, PTF11kmb, and PTF12bho. Our search revealed 1 additional object, PTF10hcw, as a candidate Ca-rich gap transient: it was reported as a $\mathrm{SN} \mathrm{Ib}$ based on the single spectrum taken (Gal-Yam et al. 2010), but also shows unusually strong $\mathrm{Ca}$ emission similar to that of SN 2005E. While we lack both a wellsampled light curve and a nebular spectrum and therefore cannot confirm that it was a Ca-rich gap transient, we show the light curve and spectrum in the Appendix. We note that PTF10hcw was found in the outskirts of NGC 2639 , offset 25 !" $7(5.9 \mathrm{kpc})$ from the nucleus, and so is an example of a possible PTF Ca-rich gap transient detected on top of its host galaxy.

The 11 remaining gap objects include PTF10bhp (a SN .Ia candidate; Kasliwal et al. 2010), one faint SN Ic, and 9 objects with hydrogen emission that are likely either LBV outbursts or luminous red novae (N. Blagorodnova et al. 2017, in preparation). Notably, these objects exhibit both comparable luminosities and similar or faster timescales than the Ca-rich gap transients, and all 11 of these transients were detected on top of their host-galaxy disks. Thus, we show that PTF is capable of detecting fast and faint transients on bright galaxy backgrounds. While the detection efficiency is likely lower in such regions of high surface brightness, the relative lack of Carich gap transients found at small offsets in PTF is unlikely to be caused solely by a bias against being able to detect them.

With the exception of SN 2012hn, which was discovered by the Catalina Real-Time Transients Survey (Drake et al. 2009) and followed up by PESSTO (Valenti et al. 2014), the remainder of the candidate Ca-rich gap transients were found by the Lick Observatory Supernova Survey (LOSS; Filippenko et al. 2001). LOSS is a galaxy-targeted survey, so that an alternative explanation for the discrepancy could be that LOSS is missing transients at large offsets, or at least is less efficient than PTF in discovering and monitoring them (although Foley (2015) argued that the LOSS field of view is sufficiently large 

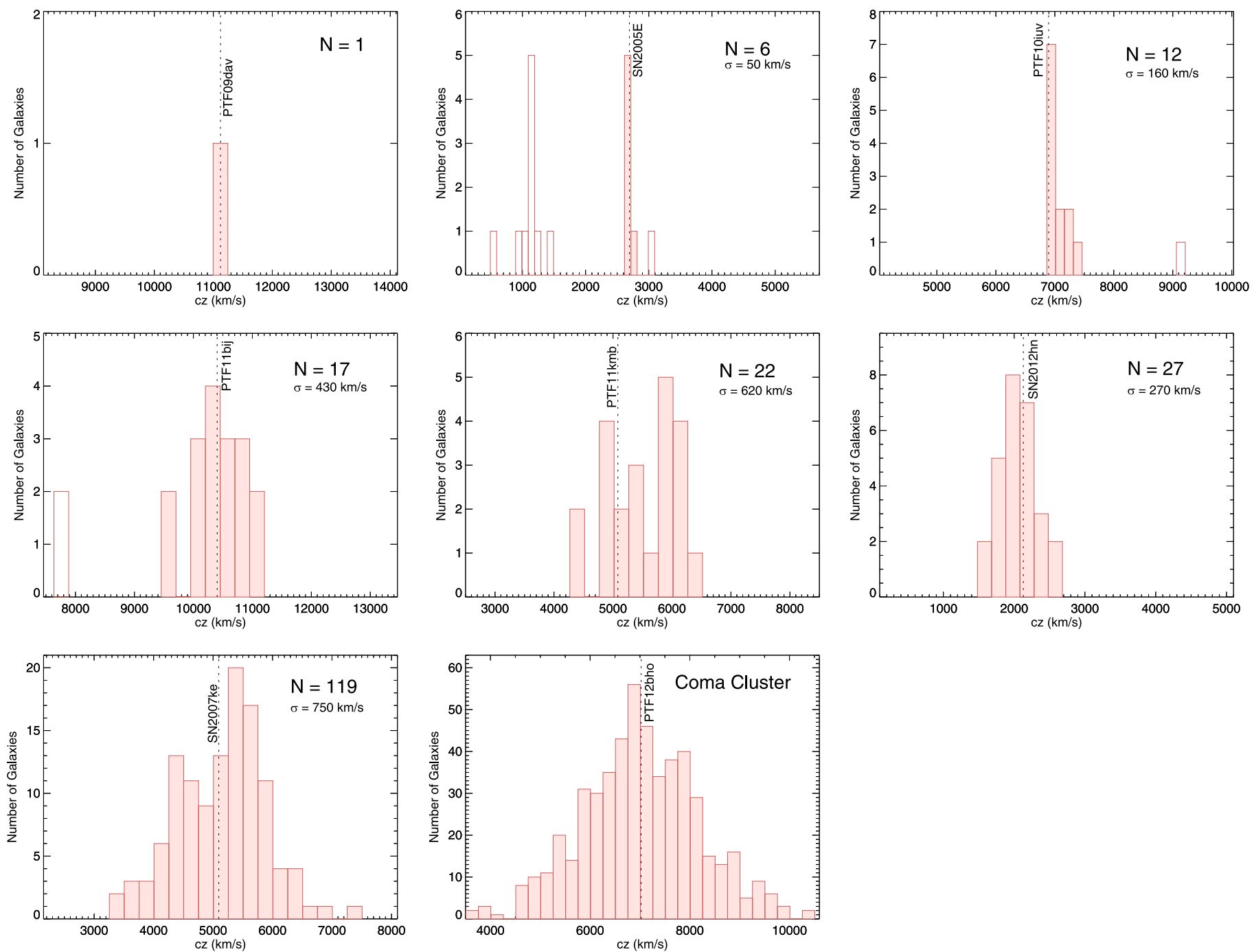

Figure 12. Radial velocity distributions of galaxies in NED within $1 \mathrm{Mpc}$ radius and $\pm 3000 \mathrm{~km} \mathrm{~s}^{-1}$ of the position of each Ca-rich gap transient. The dotted lines mark the redshifts of the (presumed) host galaxy of each transient. The shaded parts of the histograms indicate galaxies that would be group members based on the measured mean velocity and dispersion, while the unshaded part shows other galaxies along the line of sight. The number of galaxies in each group and the line-ofsight velocity dispersion is indicated in each panel. Only PTF09dav appears to come from an isolated galaxy; the remainder are in groups of various richness including two galaxy clusters (SN 2007ke and PTF12bho).

to have been able to detect the objects reported by K12). A final possibility is that not all of the candidates from LOSS belong to the class of Ca-rich gap transients: Valenti et al. (2014) point out that strong [Ca II] nebular emission can also be seen in faint core-collapse SNe (Pastorello et al. 2004) and in other peculiar Type I explosions such as SN 2008 ha (Foley et al. 2009; Valenti et al. 2009). The offset discrepancy can likely be explained by a combination of small-number statistics, contamination by other transients with strong [Ca II] in their nebular spectra, and a potential survey bias in LOSS against identifying and following up objects found far away from the targeted galaxy as SNe.

\subsection{Group and Cluster Environments}

In addition to their large offsets, it is interesting that both PTF11kmb and PTF12bho are found in group or cluster environments - both are intragroup or intracluster transients. To quantify the extent to which this is also true for other $\mathrm{Ca}$ rich gap transients, we search NED for all galaxies within $1 \mathrm{Mpc}$ and $\pm 3000 \mathrm{~km} \mathrm{~s}^{-1}$ of each Ca-rich gap transient. Group members (if any) are then determined by an iterative $3 \sigma$ clipping algorithm (Yahil \& Vidal 1977; Zabludoff \& Mulchaey 1998). We use the biweight estimators for location (central velocity) and scale (velocity dispersion) at each step (Beers et al. 1990). The resulting velocity histograms are shown in Figure 12.

Out of the $8 \mathrm{Ca}$-rich gap transients confirmed to date, only PTF09dav appears to be in a completely isolated environment, at least to the depth of galaxies with redshifts listed in NED. While NGC 1032, the host of SN 2005E, was described as an isolated galaxy by Perets et al. (2010), there are 5 companions within $\pm 150 \mathrm{~km} \mathrm{~s}^{-1}$ in a $1 \mathrm{Mpc}$ radius, making it part of a (sparse) galaxy group. PTF10iuv, PTF11bij, PTF11kmb, and SN 2012hn are all found in galaxy groups with 10-30 members listed in NED. SN 2007ke, like PTF12bho, is found in a galaxy cluster. We also note that the Ca-rich gap transients are generally found in galaxies near the center of the velocity distribution, and are in several cases also found near the brightest group or cluster galaxy.

Cluster environments are rare; finding a significant fraction of Ca-rich gap transients there is therefore unlikely to be a 
coincidence. We can use our measured line-of-sight velocity dispersions to estimate the underlying halo masses following Equation (6) in Yang et al. (2007),

$$
\sigma=397.9 \mathrm{~km} \mathrm{~s}^{-1}\left(\frac{M_{h}}{10^{14} h^{-1} M_{\odot}}\right)^{0.3214},
$$

suggesting that about half of our systems have halo masses $\gtrsim 10^{14} M_{\odot} h^{-1}$. At these masses, the halo mass function starts turning over exponentially (e.g., Press \& Schechter 1974; Tinker et al. 2008). While the numbers are low, we conclude that Ca-rich gap transients seem to show a preference for massive or dense environments.

\section{Discussion and Conclusions}

We have shown that PTF11kmb and PTF12bho are members of the class of Ca-rich gap transients as described by K12, having low peak luminosities, rapid evolution, and a rapid transition to the nebular phase with a nebular spectrum dominated by $\mathrm{Ca}$ features. The addition of these two objects brings the number of confirmed members (with well-sampled photometric and spectroscopic information) to eight. PTF11kmb and PTF12bho are also the Ca-rich gap transients found at the largest offsets from their presumed host galaxies to date, and are perhaps better described as intragroup and intracluster transients (e.g., Gal-Yam et al. 2003; Sand et al. 2011).

Taken together, the host environments of the Ca-rich gap transients are striking. The fact that any are found in early-type galaxies indicates that the progenitors are likely old. We note, however, that the observed fraction of early-type galaxies $(7 / 8)$ is much higher than what is seen for SNe Ia and sGRBs, whose host populations include $>50 \%$ late-time galaxies, indicating that their rates depend on both the star formation rate and stellar mass (Sullivan et al. 2006; Fong et al. 2013). Moreover, if the rate of Ca-rich gap transients depended only on stellar mass, we would expect their host-galaxy demographics to follow the distribution of stellar mass in the local universe, and thus find about equal numbers of late-type and early-type hosts (Kochanek et al. 2001; Bell et al. 2003). The fact that the overwhelming majority of hosts are early-type galaxies therefore points to other factors being important for the progenitor population.

Given the preference for early-type galaxies and for group and cluster environments, a leading candidate for the dominant factor is stellar population age. In general, massive halos like the ones that host clusters and massive groups formed the majority of their stars before $z=2$ (e.g., Behroozi et al. 2013), and are therefore dominated by stellar populations older than $>10 \mathrm{Gyr}$. If this is the driving factor for the host-galaxy preference, it implies that the progenitor systems for Ca-rich gap transients have a very long merging time (or more generally, a delay-time distribution that peaks at long timescales). These massive environments are also where the greatest proportion of stars are found in the intracluster light (ICL), which may at least partially explain the remote environments (Gonzalez et al. 2005; Mihos et al. 2005, 2013; Behroozi et al. 2013). If age is indeed the driving factor, however, we would expect to also find a fraction within elliptical or S0 galaxies. We note that there are at least two such examples among the candidate Ca-rich gap transients: SN 2005cz in the elliptical galaxy NGC 4589, and SN 2000ds in NGC 2768 (Filippenko et al. 2003; Kawabata et al. 2010; Perets et al. 2011).
Our search of the PTF database demonstrates that the relative lack of Ca-rich gap transients at small offsets found in PTF is not likely to be caused by a selection effect, and rather illustrates the importance of wide-field, galaxy-untargeted surveys in detecting such remote transients. One proposed explanation for the offset distribution has been that Ca-rich gap transients originate in very faint systems such as globular clusters, in which case a preference toward group and cluster environments is also predicted (Yuan et al. 2013; Sell et al. 2015). However, the deep photometric limits at the locations of Ca-rich gap transients, including those presented in this paper for SN 2005E and PTF12bho, rule out an underlying dwarf galaxy as well as most of the globular cluster luminosity function. While our HST imaging does not rule out a globular cluster origin for PTF $11 \mathrm{kmb}$, it is unlikely to be a dominant channel for producing Ca-rich gap transients, at least assuming they are not kicked out of the globular cluster before merging.

The general lack of underlying or faint host systems is consistent with previous findings (Lyman et al. 2014, 2016), and has been used to argue that Ca-rich gap transients do not form "in situ" at these remote locations, but have traveled to their explosion sites. One attractive property of the NS-WD merger scenario (Metzger 2012; Sell et al. 2015; Margalit \& Metzger 2016) is that the binary would have received a kick in the SN explosion that creates the neutron star; such SN kicks are indeed also evoked to explain the offset distribution of sGRBs, which is consistent with theoretical predictions for NS-NS mergers (Fong \& Berger 2013). In this context, it is interesting to note that the offset distribution for Ca-rich gap transients is significantly more extreme than that of sGRBs (Section 4.2; Figure 11). In addition, stellar population synthesis models predict lower median velocities for WD-NS systems than for NS-NS systems (Behroozi et al. 2014). If the origin of the large offsets is SN kicks, then the merger times for Ca-rich gap transients must accordingly be significantly longer than for sGRBs.

The offset-based argument only holds if we assume that the Ca-rich gap transients originate from the galaxies and thus need to travel a significant distance before they explode. We note, however, that if the progenitor systems are formed in globular clusters, a SN kick could easily eject the system, since the escape velocities of globular clusters are only on the order of tens of $\mathrm{km} \mathrm{s}^{-1}$ (e.g., Gnedin et al. 2002). This means that if the progenitors are NS-WD binaries, the lack of underlying host systems does not necessarily rule out a contribution from systems originating in globular clusters. In fact, simulations of globular clusters show that in order to match the rate of observed low-mass X-ray binaries, they must assume that a fraction of neutron stars form in electron-capture SNe since most of the neutron star systems forming in core-collapse SNe end up being ejected from the cluster (Ivanova et al. 2008). A caveat is that only binaries formed on timescales shorter than the time to the SN creating the neutron star would be ejected this way. We would still expect that binaries that formed dynamically after the neutron star is created will explode within the globular cluster.

If the progenitor system is not a NS-WD binary but rather a WD-WD binary (e.g., Perets et al. 2010; Waldman et al. 2011; Sim et al. 2012; Dessart \& Hillier 2015), then the remote locations require a different origin than $\mathrm{SN}$ kicks. One possibility, as noted above, is that group and cluster environments have a large proportion of stars in ICL, resulting from galaxy-galaxy stripping and galaxy mergers in the formation of the cluster. The case for a globular cluster origin, 
on the other hand, is weaker without being able to invoke SN kicks, but in principle a WD-WD binary could be ejected from a globular cluster through dynamical interactions. A third possibility was proposed by Foley (2015), who suggested that Ca-rich gap transients originate in nuclear regions of their host galaxies and are flung out at high velocities following an interaction with the central supermassive black hole. At the very least, any progenitor model will have to be able to explain not just the properties of the Ca-rich transients themselves, but also their strong environment preferences.

Finally, we note that the Ca-rich gap transients constitute one emerging subclass among a number of peculiar transients that have been discovered over the past decade and that are some combination of faint, fast, and hydrogen-poor, or they are found in old environments and at large offsets (e.g., Foley et al. 2010, 2013; Kasliwal et al. 2010; Maguire et al. 2011; Sanders et al. 2013; Inserra et al. 2015). While the properties described by K12 were modeled on SN 2005E and attempted to define the maximum set of characteristics that give the largest number of related transients, they were still empirically defined, therefore it is possible that they do not all constitute the same physical phenomenon. Notably, K12 allowed for diversity in the photospheric-phase spectra and did not require the detection of $\mathrm{He}$, without which PTF09dav would not have been considered a member of this class. The fact that PTF09dav seems to be an outlier both in terms of its SN and host-galaxy properties suggests that it may have a distinct physical origin from the rest of the objects in the sample. Similarly, while we argue here that PTF12bho should be considered a Ca-rich gap transient at least as described in K12, its photospheric spectrum is unique among the objects discovered to date. While sample sizes are currently small, upcoming surveys like the Zwicky Transient Facility and the Large Synoptic Survey Telescope should continue to discover more of these rare transients, shedding light on their true origins.

R.L. thanks Andrew Wetzler, Wen-fai Fong, Mark Sullivan, Dan Milisavljevic, Giorgos Leloudas, Jesper Sollerman, and Ryan Chornock for useful discussions and acknowledges helpful interactions with Lars Bildsten, Eliot Quataert, and Dan Kasen at a PTF Theory Network retreat funded by the Gordon and Betty Moore Foundation through Grant GBMF5076. We thank J. Silverman, B. Dilday, J. Bloom, B. Sesar, D. Levitan, P. Groot, D. Perley, A. Horesh, K. Mooley, and D. Xu for assisting with the observations presented in this paper. The Intermediate Palomar Transient Factory project is a scientific collaboration among the California Institute of Technology, Los Alamos National Laboratory, the University of WisconsinMilwaukee, the Oskar Klein Center, the Weizmann Institute of Science, the TANGO Program of the University System of Taiwan, and the Kavli Institute for the Physics and Mathematics of the Universe. Support for HST Program GO-13864 was provided by NASA through a grant from the Space Telescope Science Institute, which is operated by the AURA, Inc., under NASA contract NAS 5-26555. We thank F. Yuan, M. Sullivan, D. Perley, R. M. Quimby, and S. B. Cenko for their contributions to the HST proposal. This work was supported by the GROWTH project funded by the National Science Foundation under Grant 1545949. The National Energy Research Scientific Computing Center, which is supported by the Office of Science of the U.S. Department of Energy under Contract No. DEAC02-05CH11231, provided staff, computational resources, and

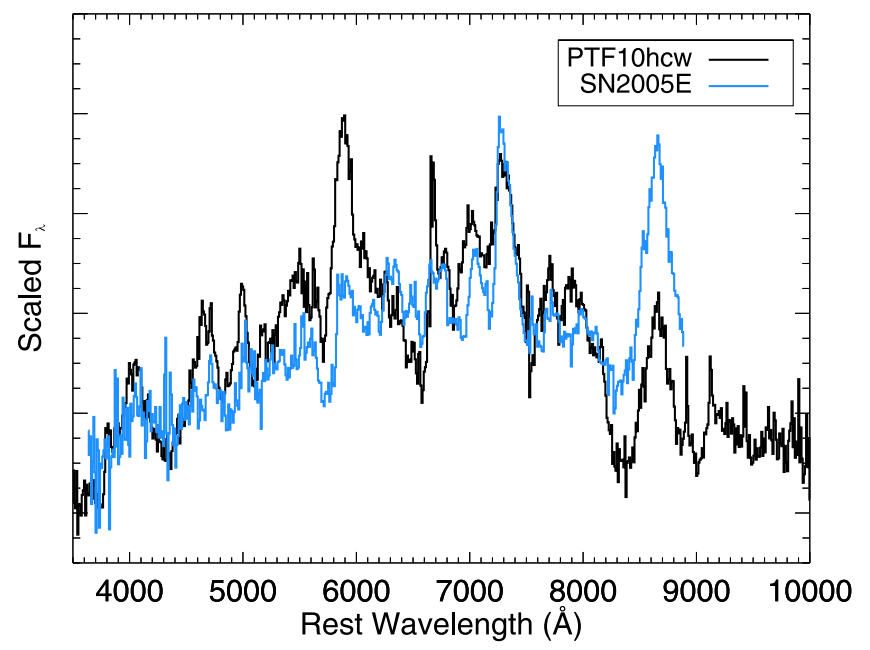

Figure 13. Our single spectrum of PTF10hcw (black), compared to SN 2005E (blue). The PTF10hcw spectrum is taken at phase 13 days past discovery (and 18 days after the last nondetection), while the SN 2005E spectrum is at phase 24 days past discovery. Like SN 2005E, PTF10hcw developed [Ca II] $\lambda \lambda$ 7291,7324 emission at an early phase.

data storage for this project. A.G.-Y. is supported by the EU/ FP7 via ERC grant No. 307260, the Quantum Universe I-Core program by the Israeli Committee for planning and funding, and the ISF, Minerva and ISF grants, WIS-UK "making connections," and Kimmel and YeS awards. A.V.F. is grateful for financial support from the Christopher R. Redlich Fund, the TABASGO Foundation, and NSF grant AST-1211916. D.A.H. and C.M. are supported by NSF grant AST-313484. This research has made use of the NASA/IPAC Extragalactic Database (NED), which is operated by the Jet Propulsion Laboratory, California Institute of Technology, under contract with the National Aeronautics and Space Administration. Some of the data presented herein were obtained at the W.M. Keck Observatory, which is operated as a scientific partnership among the California Institute of Technology, the University of California and the National Aeronautics and Space Administration. The Observatory was made possible by the generous financial support of the W.M. Keck Foundation. The authors wish to recognize and acknowledge the very significant cultural role and reverence that the summit of Mauna Kea has always had within the indigenous Hawaiian community. We are most fortunate to have the opportunity to conduct observations from this mountain.

Facilities: PO:1.2m, PO:1.5m, PO:Hale, Keck:I, Keck:II, Subaru, HST.

\section{Appendix \\ Ca-rich Gap Transient Candidate PTF10hcw}

The object PTF10hcw was discovered in P48 data at (J2000) $\alpha=08^{\mathrm{h}} 43^{\mathrm{m}} 36^{\mathrm{s}} .22, \delta=+50^{\circ} 12^{\prime} 38^{\prime \prime} .5$ on 2010 May 02.2 , at a discovery magnitude of $R=19.3 \mathrm{mag}$. No emission was detected at this location to a limit $R>20.3$ mag on 2010 April 27. The object was classified as a $\mathrm{SN} \mathrm{Ib}$ based on an LRIS spectrum taken on 2010 May 15 (Gal-Yam et al. 2010), which is shown in Figure 13.

We suggest that PTF10hcw may have been a Ca-rich gap transient based on the following two observations. First, the spectrum shows emerging [Ca II] emission, which is not present in normal SNe Ib at such an early phase (e.g., Liu et al. 2016), but was also seen in SN 2005E. Second, while the light curve 


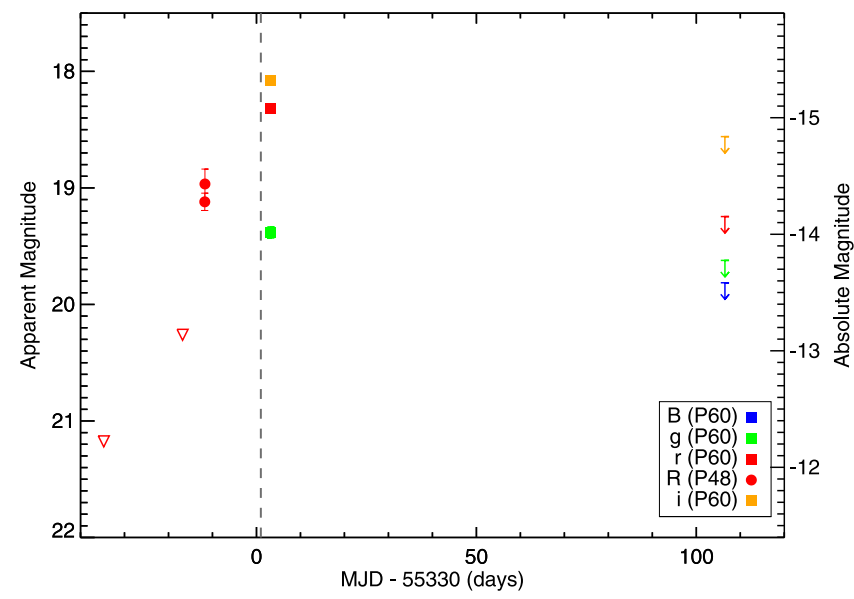

Figure 14. Light curve of PTF10hcw. Triangles and arrows show upper limits from P48 and P60, respectively. The light curve is extremely sparse as the field went into solar conjunction shortly after the transient was discovered, hence the 100 day gap. Neither the rise time nor the peak magnitude are very well constrained, but based on the available data, it is likely that the peak magnitude is comparable to that of the Ca-rich gap transients (Figure 4). The time of the spectrum displayed in Figure 13 is shown by the dashed line.

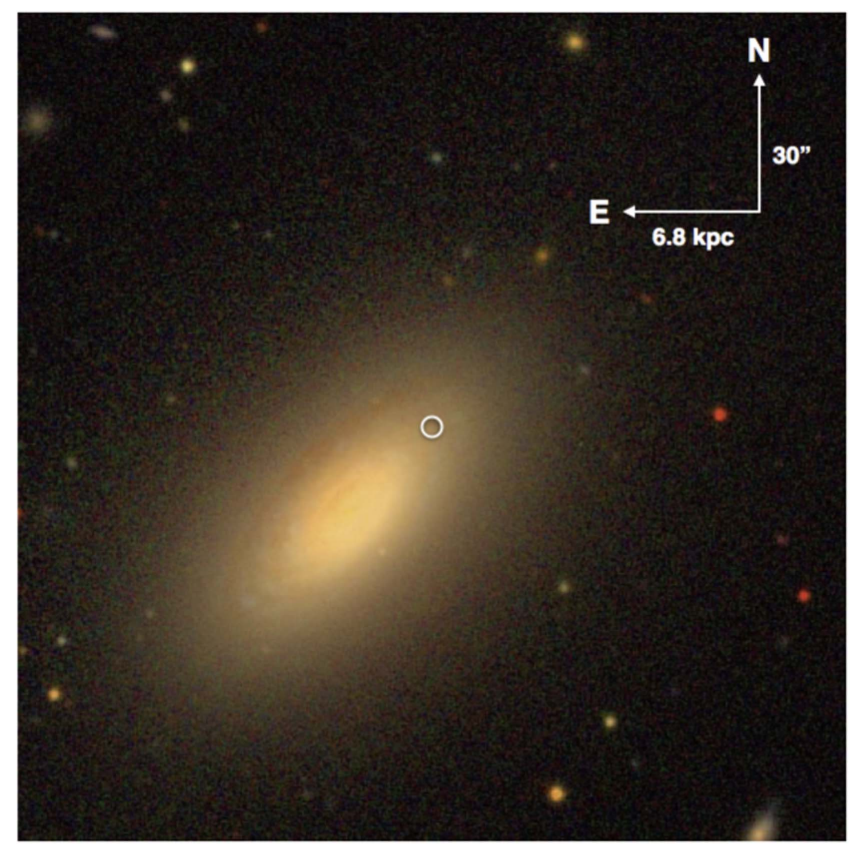

Figure 15. $3^{\prime} \times 3^{\prime}$ composite gri SDSS image of NGC 2639, the host galaxy of PTF10hcw. The image is centered on the location of the transient, which is shown by the white circle.

(shown in Figure 14) for this object is extremely sparse since it was discovered as the field was going into solar conjunction, the brightest observed $r$-band magnitude was $M_{r}=$ $-15.1 \mathrm{mag}$. This was at a phase 15 days after discovery (and 21 days after the last nondetection), so it is likely that the SN was near peak light, and by extension that the peak brightness was fainter than that of a typical SN Ib. This is, in itself, not enough to classify PTF10hcw as a Ca-rich gap transient as opposed to a peculiar SN Ib, as we lack both a well-sampled light curve and a nebular-phase spectrum; we merely present it here as a candidate.
We note that PTF10hcw was found at a projected offset 25 !.7 (5.9 kpc) from the nucleus of NGC 2639, which is classified as an Sa galaxy with LINER nuclear activity in NED. The host galaxy and SN location are shown in Figure 15. NGC 2639 is the brightest galaxy in a group of seven members with redshifts in NED, and with a line-of-sight velocity dispersion of $130 \mathrm{~km} \mathrm{~s}^{-1}$.

\section{References}

Arnett, W. D. 1982, ApJ, 253, 785

Baron, E., Hauschildt, P. H., Nugent, P., \& Branch, D. 1996, MNRAS, 283, 297

Beckwith, S. V. W., Stiavelli, M., Koekemoer, A. M., et al. 2006, AJ, 132, 1729

Beers, T. C., Flynn, K., \& Gebhardt, K. 1990, AJ, 100, 32

Behroozi, P. S., Ramirez-Ruiz, E., \& Fryer, C. L. 2014, ApJ, 792, 123

Behroozi, P. S., Wechsler, R. H., \& Conroy, C. 2013, ApJ, 770, 57

Bell, E. F., McIntosh, D. H., Katz, N., \& Weinberg, M. D. 2003, ApJS, 149, 289

Berger, E. 2010, ApJ, 722, 1946

Bloom, J. S., Kulkarni, S. R., \& Djorgovski, S. G. 2002, AJ, 123, 1111

Branch, D., Benetti, S., Kasen, D., et al. 2002, ApJ, 566, 1005

Brown, T. M., Baliber, N., Bianco, F. B., et al. 2013, PASP, 125, 1031

Burrows, D. N., Hill, J. E., Nousek, J. A., et al. 2005, Space Sci. Rev., 120, 165

Cenko, S. B., Fox, D. B., Moon, D.-S., et al. 2006, PASP, 118, 1396

Dessart, L., \& Hillier, D. J. 2015, MNRAS, 447, 1370

Drake, A. J., Djorgovski, S. G., Mahabal, A., et al. 2009, ApJ, 696, 870

Faber, S. M., Phillips, A. C., Kibrick, R. I., et al. 2003, Proc. SPIE, 4841, 1657

Filippenko, A. V. 1997, ARA\&A, 35, 309

Filippenko, A. V., Chornock, R., Swift, B., et al. 2003, IAU Circ., 8159, 2

Filippenko, A. V., Li, W. D., Treffers, R. R., \& Modjaz, M. 2001, in ASP Conf. Ser. 246, IAU Coll. 183: Small Telescope Astronomy on Global Scales, ed. B. Paczynski, W.-P. Chen, \& C. Lemme (San Francisco, CA: ASP), 121

Foley, R. J. 2015, MNRAS, 452, 2463

Foley, R. J., Challis, P. J., Chornock, R., et al. 2013, ApJ, 767, 57

Foley, R. J., Chornock, R., Filippenko, A. V., et al. 2009, AJ, 138, 376

Foley, R. J., Narayan, G., Challis, P. J., et al. 2010, ApJ, 708, 1748

Fong, W., \& Berger, E. 2013, ApJ, 776, 18

Fong, W., Berger, E., Chornock, R., et al. 2013, ApJ, 769, 56

Fong, W., Berger, E., \& Fox, D. B. 2010, ApJ, 708, 9

Fremling, C., Sollerman, J., Taddia, F., et al. 2016, A\&A, 593, A68

Gal-Yam, A., Arcavi, I., Ami, S. B., et al. 2010, ATel, 2631

Gal-Yam, A., Maoz, D., Guhathakurta, P., \& Filippenko, A. V. 2003, AJ, 125,1087

Gal-Yam, A., Xu, D., Ben-Ami, S., et al. 2011, ATel, 3631

Gnedin, O. Y., Zhao, H., Pringle, J. E., et al. 2002, ApJL, 568, L23

Gonzalez, A. H., Zabludoff, A. I., \& Zaritsky, D. 2005, ApJ, 618, 195

Inserra, C., Sim, S. A., Wyrzykowski, L., et al. 2015, ApJL, 799, L2

Ivanova, N., Heinke, C. O., Rasio, F. A., Belczynski, K., \& Fregeau, J. M. 2008, MNRAS, 386, 553

Jordán, A., McLaughlin, D. E., Côté, P., et al. 2007, ApJS, 171, 101

Kasliwal, M. M. 2012, PASA, 29, 482

Kasliwal, M. M., Kulkarni, S. R., Gal-Yam, A., et al. 2010, ApJL, 723, L98

Kasliwal, M. M., Kulkarni, S. R., Gal-Yam, A., et al. 2012, ApJ, 755, 161

Kawabata, K. S., Maeda, K., Nomoto, K., et al. 2010, Natur, 465, 326

Kochanek, C. S., Pahre, M. A., Falco, E. E., et al. 2001, ApJ, 560, 566

Koda, J., Yagi, M., Yamanoi, H., \& Komiyama, Y. 2015, ApJL, 807, L2

Komatsu, E., Smith, K. M., Dunkley, J., et al. 2011, ApJS, 192, 18

Law, N. M., Dekany, R. G., Rahmer, G., et al. 2010, Proc. SPIE, 7735, $77353 \mathrm{M}$

Law, N. M., Kulkarni, S. R., Dekany, R. G., et al. 2009, PASP, 121, 1395

Liu, Y.-Q., Modjaz, M., Bianco, F. B., \& Graur, O. 2016, ApJ, 827, 90

Lucy, L. B. 1991, ApJ, 383, 308

Lyman, J. D., James, P. A., Perets, H. B., et al. 2013, MNRAS, 434, 527

Lyman, J. D., Levan, A. J., Church, R. P., Davies, M. B., \& Tanvir, N. R. 2014, MNRAS, 444, 2157

Lyman, J. D., Levan, A. J., James, P. A., et al. 2016, MNRAS, 458, 1768

Maguire, K., Sullivan, M., Thomas, R. C., et al. 2011, MNRAS, 418, 747

Margalit, B., \& Metzger, B. D. 2017, MNRAS, 465, 2790

Masci, F. J., Laher, R. R., Rebbapragada, U. D., et al. 2017, PASP, 129 , 014002

Metzger, B. D. 2012, MNRAS, 419, 827 
Mihos, J. C., Harding, P., Feldmeier, J., \& Morrison, H. 2005, ApJL, 631, L41 Mihos, J. C., Harding, P., Rudick, C. S., \& Feldmeier, J. J. 2013, ApJL, 764, L20

Mulchaey, J. S., Kasliwal, M. M., \& Kollmeier, J. A. 2014, ApJL, 780, L34

Oke, J. B., Cohen, J. G., Carr, M., et al. 1995, PASP, 107, 375

Oke, J. B., \& Gunn, J. E. 1982, PASP, 94, 586

Pastorello, A., Zampieri, L., Turatto, M., et al. 2004, MNRAS, 347, 74

Perets, H. B., Gal-yam, A., Crockett, R. M., et al. 2011, ApJL, 728, L36

Perets, H. B., Gal-Yam, A., Mazzali, P. A., et al. 2010, Natur, 465, 322

Press, W. H., \& Schechter, P. 1974, ApJ, 187, 425

Rahmer, G., Smith, R., Velur, V., et al. 2008, Proc. SPIE, 7014, 70144Y

Rau, A., Kulkarni, S. R., Law, N. M., et al. 2009, PASP, 121, 1334

Roming, P. W. A., Kennedy, T. E., Mason, K. O., et al. 2005, Space Sci. Rev., 120,95

Sand, D. J., Graham, M. L., Bildfell, C., et al. 2011, ApJ, 729, 142

Sanders, N. E., Soderberg, A. M., Foley, R. J., et al. 2013, ApJ, 769, 39

Schlafly, E. F., \& Finkbeiner, D. P. 2011, ApJ, 737, 103
Sell, P. H., Maccarone, T. J., Kotak, R., Knigge, C., \& Sand, D. J. 2015, MNRAS, 450, 4198

Sim, S. A., Fink, M., Kromer, M., et al. 2012, MNRAS, 420, 3003

Sullivan, M., Kasliwal, M. M., Nugent, P. E., et al. 2011, ApJ, 732, 118

Sullivan, M., Le Borgne, D., Pritchet, C. J., et al. 2006, ApJ, 648, 868

Thomas, R. C., Nugent, P. E., \& Meza, J. C. 2011, PASP, 123, 237

Tinker, J., Kravtsov, A. V., Klypin, A., et al. 2008, ApJ, 688, 709

Valenti, S., Howell, D. A., Stritzinger, M. D., et al. 2016, MNRAS, 459, 3939

Valenti, S., Pastorello, A., Cappellaro, E., et al. 2009, Natur, 459, 674

Valenti, S., Yuan, F., Taubenberger, S., et al. 2014, MNRAS, 437, 1519

Waldman, R., Sauer, D., Livne, E., et al. 2011, ApJ, 738, 21

Yagi, M., Koda, J., Komiyama, Y., \& Yamanoi, H. 2016, ApJS, 225, 11

Yahil, A., \& Vidal, N. V. 1977, ApJ, 214, 347

Yang, X., Mo, H. J., van den Bosch, F. C., et al. 2007, ApJ, 671, 153

Yuan, F., Kobayashi, C., Schmidt, B. P., et al. 2013, MNRAS, 432, 1680

Zabludoff, A. I., \& Mulchaey, J. S. 1998, ApJ, 496, 39 